\title{
QUÍMICA ATMOSFÉRICA DO ENXOFRE (IV): EMISSÕES, REAÇÕES EM FASE AQUOSA E IMPACTO AMBIENTAL
}

Cláudia Rocha Martins e Jailson Bittencourt de Andrade*

Instituto de Química, Universidade Federal da Bahia, 40170290 Salvador - BA

Recebido em 7/7/00; aceito em 31/10/01

ATMOSPHERIC CHEMISTRY OF SULFUR (IV): EMISSIONS, AQUEOUS PHASE REACTIONS AND ENVIRONMENTAL EFFECTS. The oxidation process of sulfur(IV) species by oxygen, ozone and nitrogen oxides, catalysed by trace metal ions, can play an important role in atmospheric chemistry processes like acid rain, visibility degradation and health hazard. An overview of the more relevant investigations on emissions sources, aqueous phase conversion process and environmental impact is presented.

Keywords: sulphur dioxide; sulfite; atmospheric chemistry of sulfur (IV).

\section{INTRODUÇÃO}

Dióxido de enxofre, um dos mais comuns poluentes atmosféricos, é introduzido no ambiente em grandes quantidades, proveniente tanto de fontes antropogênicas quanto de fontes naturais. Uma vez emitido, $\mathrm{SO}_{2}(\mathrm{~g})$ pode reagir com vários oxidantes presentes na atmosfera e formar sulfato particulado, na forma de gotas de $\mathrm{H}_{2} \mathrm{SO}_{4}$ ou na forma de partículas neutralizadas tais como sulfato de amônio. $\mathrm{O}$ processo de oxidação do $\mathrm{SO}_{2}$ e outras espécies de $\mathrm{S}$ (IV) ocorre tanto em fase gasosa em dias claros, como também em fase aquosa, na presença de nuvens e nevoeiros. Tal processo, além de resultar na formação de sulfato particulado, contribui significativamente para a produção de acidez, comprometendo sobremaneira a qualidade das condições ambientais.

A oxidação via radical hidroxila tem sido considerada como a principal reação responsável pela conversão homogênea (fase gasosa) do $\mathrm{SO}_{2}{ }^{1}$. Já a conversão do $\mathrm{S}(\mathrm{IV})$ a sulfato, que ocorre na fase aquosa atmosférica, apresenta uma química mais complexa e dependente de um maior número de variáveis, tais como: concentração de diversas espécies oxidantes $\left(\mathrm{H}_{2} \mathrm{O}_{2}, \mathrm{O}_{3}, \mathrm{NO}_{\mathrm{x}}\right)$, presença de íons metálicos, tamanho e composição da gota ${ }^{1}$. Uma terceira rota, citada para a oxidação do $\mathrm{S}(\mathrm{IV})$ na atmosfera, é a adsorção do $\mathrm{SO}_{2}$ gasoso em superfícies sólidas e a subsequente reação com oxidantes adsorvidos na partícula ${ }^{1-3}$. A contribuição relativa do processo heterogêneo em superfícies é, no entanto, bem menor quando comparada com os processos de conversão por radical hidroxila ou em solução aquosa.

Estudos em campo indicam que a importância do processo de conversão homogênea (fase gasosa) e heterogênea (fase líquida) depende de uma variedade de fatores climatológicos, tais como umidade relativa e intensidade da radiação solar incidente ${ }^{4}$. O acompanhamento dos processos atmosféricos de conversão do S(IV), por um período de quatro meses, na região de Heidelberg, sugeriu que a metade do sulfato formado no ar atmosférico é proveniente do processo de conversão homogênea, enquanto que cerca da outra metade é devida à conversão heterogênea, que ocorre em nuvens e nevoei$\operatorname{ros}^{4,5}$. Após um episódio de formação de neblina, em Los Angeles, altas concentrações de sulfato foram observadas no aerossol. A simulação do episódio envolvendo reação em fase gasosa e fase líquida, indica que cerca de $40 \%$ da concentração de sulfato deve ser

\footnotetext{
* e-mail: jailsong@ufba.br
}

proveniente do processo homogêneo, enquanto que as diversas reações possíveis de ocorrerem em fase aquosa, devem ser responsáveis por cerca de $60 \%$ do sulfato produzido ${ }^{6}$. Resultados semelhantes foram encontrados em simulações de oxidação do $\mathrm{SO}_{2}$ em atmosferas marinhas ${ }^{7}$. Simulações da oxidação do $\mathrm{SO}_{2}$ atmosférico indicam ainda que a reação de conversão em fase gasosa é predominante durante o dia, enquanto que à noite a produção de sulfato é devida, principalmente, às possíveis reações em fase aquosa ${ }^{8}$.

As várias reações que podem contribuir para a oxidação do $\mathrm{SO}_{2}$ a sulfato em fase aquosa têm sido estudadas por muitos investigadores ${ }^{9-15}$. A oxidação por $\mathrm{H}_{2} \mathrm{O}_{2}$ parece ser a mais favorável devido à alta velocidade de reação, porém na ausência de concentrações significativas de $\mathrm{H}_{2} \mathrm{O}_{2}$ na atmosfera, a oxidação por ozônio ou por oxigênio catalisada por metais de transição pode se tornar significante ${ }^{1,14}$.

A maioria dos estudos realizados em laboratório, sobre a oxidação de S(IV) catalisada por metais, tem focalizado as reações em solução aquosa em presença de $\mathrm{O}_{2}$ e dos íons metálicos $\mathrm{Mn}$ (II), $\mathrm{Fe}(\mathrm{III}), \mathrm{Cu}$ (II) e $\mathrm{Co}$ (II) ou na interação entre oxigênio e sulfitos ${ }^{9,16}$. Apesar do grande número de estudos sobre a oxidação de espécies de S(IV) catalisada por metais, existe discussão na literatura com relação a quais íons metálicos são os catalisadores mais importantes na atmosfera ${ }^{12,17-26}$. As discordâncias podem ser observadas nos diferentes valores de constantes de velocidade, de energias de ativação e na dependência com o pH, para as reações estudadas ${ }^{9,26}$.

Relativamente poucos estudos foram realizados até o momento, sobre a influência catalítica dos íons metálicos em presença de ozônio, ou em presença de $\mathrm{NO}_{2}$, na oxidação de $\mathrm{S}(\mathrm{IV})$ em solução aquosa. Investigações envolvendo $\mathrm{Fe}(\mathrm{III}), \mathrm{Fe}(\mathrm{II}), \mathrm{Mn}$ (II) e $\mathrm{Cu}(\mathrm{II})$ foram conduzidas na presença de ozônio, porém, mais uma vez, os resultados apresentados se mostram controvertidos com relação a quais íons são catalisadores para a reação ${ }^{11,14,27}$. $\mathrm{O}$ mesmo tem sido observado para as espécies metálicas na presença de $\mathrm{NO}_{2}$. Enquanto alguns resultados mostram que o $\mathrm{Fe}$ (II) exibe forte efeito catalítico ${ }^{28}$, outros não indicam este efeito para nenhum íon metálico, nas reações em estudo $^{29}$.

\section{EMISSÕES E PROCESSOS ATMOSFÉRICOS}

\section{Compostos de enxofre}

Os compostos de enxofre mais importantes, presentes na atmosfera, compreendem espécies tais como: $\mathrm{COS}, \mathrm{CS}_{2},\left(\mathrm{CH}_{3}\right)_{2} \mathrm{~S}, \mathrm{H}_{2} \mathrm{~S}$, 
$\mathrm{SO}_{2}$ e $\mathrm{SO}_{4}^{2-}$. A transformação destes compostos na troposfera exibe uma clara tendência em direção aos estados de oxidação mais altos (Figura 1) .

\begin{tabular}{|c|c|}
\hline Estado de oxidação & Espécie \\
\hline+6 & $\underset{\uparrow}{\mathrm{H}_{2} \mathrm{SO}_{4}, \mathrm{XSO}_{4}}$ \\
\hline+4 & $\underset{\uparrow}{\mathbf{S O}_{2}}$ \\
\hline+2 & $\begin{array}{c}\text { SO } \\
\uparrow\end{array}$ \\
\hline+1 & $\begin{array}{c}\text { HSO } \\
\uparrow\end{array}$ \\
\hline $\mathbf{0}$ & $\begin{array}{l}\mathbf{S} \\
\uparrow\end{array}$ \\
\hline-1 & $\begin{array}{c}\text { SH } \\
\uparrow\end{array}$ \\
\hline-2 & $\mathrm{H}_{2} \mathrm{~S}, \mathrm{COS}, \mathrm{CS}_{2}$ \\
\hline
\end{tabular}

Figura 1. Transformação de espécies de enxofre na troposfera (adaptado da Ref. 9)

Há muitas incertezas com relação às fontes, reações e destino das espécies de enxofre na atmosfera. Os compostos de enxofre entram na atmosfera em grande parte através da atividade humana, principalmente na forma de $\mathrm{SO}_{2}$. As principais fontes antropogênicas de emissão deste gás são a queima de combustíveis fósseis e as atividades industriais, tais como o refino do petróleo, indústria de cimento, metalurgia, enquanto que a atividade vulcânica é a principal fonte responsável pelas emissões naturais de $\mathrm{SO}_{2}$. A queima da biomassa também tem sido considerada uma importante fonte de enxofre atmosférico em regiões continentais, especialmente nos trópicos. A Figura 2 mostra as principais fontes de emissão e deposição das espécies de enxofre na natureza ${ }^{30}$.

As emissões globais de $\mathrm{SO}_{2}$ são estimadas na faixa de 130-180 x $10^{12} \mathrm{~g}$ de S ano $^{-1(9)}$. De uma maneira geral a emissão antropogênica de poluentes no hemisfério Norte é muito mais alta do que no hemis- fério Sul. Enquanto que mais de $60 \%$ dos compostos de enxofre emitidos no hemisfério Norte são provenientes de fontes antropogênicas, este número cai para $7 \%$ no hemisfério Sul. Outras importantes fontes de enxofre são constituídas pelo aerossol marinho $\left(\mathrm{SO}_{4}^{2-}, 90-270 \times 10^{12} \mathrm{~g}\right.$ de S ano $\left.{ }^{-1}\right)$ e por espécies provenientes da atividade bacteriana $\left(\mathrm{H}_{2} \mathrm{~S}, 100-280 \times 10^{12} \mathrm{~g} \mathrm{de} \mathrm{S}\right.$ ano ${ }^{-1} ;\left(\mathrm{CH}_{3}\right)_{2} \mathrm{~S}$, 3-32 x $10^{12} \mathrm{~g}_{\text {de S ano }}^{-1}$; COS, 0,1-2,5 x $10^{12} \mathrm{~g}^{\mathrm{de} \mathrm{S}}$ ano $^{-1}$; $\mathrm{CS}_{2}, 0,2-$

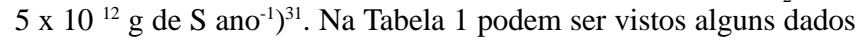
relativos à emissão natural e antropogênica de compostos de enxofre, para os hemisférios Norte e $\mathrm{Sul}^{32}$.

Tabela 1. Estimativa de emissões globais de $\mathrm{S}$ atmosférico de origem natural e antropogênica $\left(\mathrm{g} \text { de } \mathrm{S} \text { ano }{ }^{-1}\right)^{32}$

\begin{tabular}{lcc}
\hline Fontes & Hemisfério Norte & Hemisfério Sul \\
\hline Vulcões & $3 \times 10^{12}$ & $2 \times 10^{12}$ \\
Aerossol marinho & $19 \times 10^{12}$ & $25 \times 10^{12}$ \\
Biogênico & $54 \times 10^{12}$ & $44 \times 10^{12}$ \\
Natural total & $76 \times 10^{12}$ & $71 \times 10^{12}$ \\
Antropogênico total & $98 \times 10^{12}$ & $6 \times 10^{12}$ \\
\hline
\end{tabular}

Quando se compara fontes naturais e antropogênicas, deve ser mencionado que fontes naturais são globalmente distribuídas, enquanto que fontes antropogênicas são concentradas em áreas populosas. Dessa maneira, a concentração individual dos poluentes depende da área na qual a medida foi feita. Na Tabela 2 estão colocados alguns dados relativos às medidas de concentrações atmosféricas de $\mathrm{SO}_{2}$ em várias localidades.

Tabela 2. Concentrações atmosféricas de $\mathrm{SO}_{2}$

\begin{tabular}{lcc}
\hline Local/ano & Concentração $\left(\mu \mathrm{g} \mathrm{m}^{-3}\right)$ & Ref. \\
\hline Londres (ING)/1982-1984 & $44-49$ (verão) & 33 \\
& $71-77$ (inverno) & 33 \\
Nova York (EUA)/1984 & 64 & 34 \\
Rio de Janeiro (Brasil)/1985 & $4,2-74,2$ & 35 \\
Cubatão (Brasil)/1981-1990 & $18-87$ & 36 \\
\hline
\end{tabular}

Considerando a média global de emissão de compostos de enxofre para a atmosfera, verifica-se um crescimento no período compreendido entre 1850 e $1990^{37}$. Após a Segunda Guerra Mundial, os aumentos mais rápidos em emissões foram registrados na Ásia, África

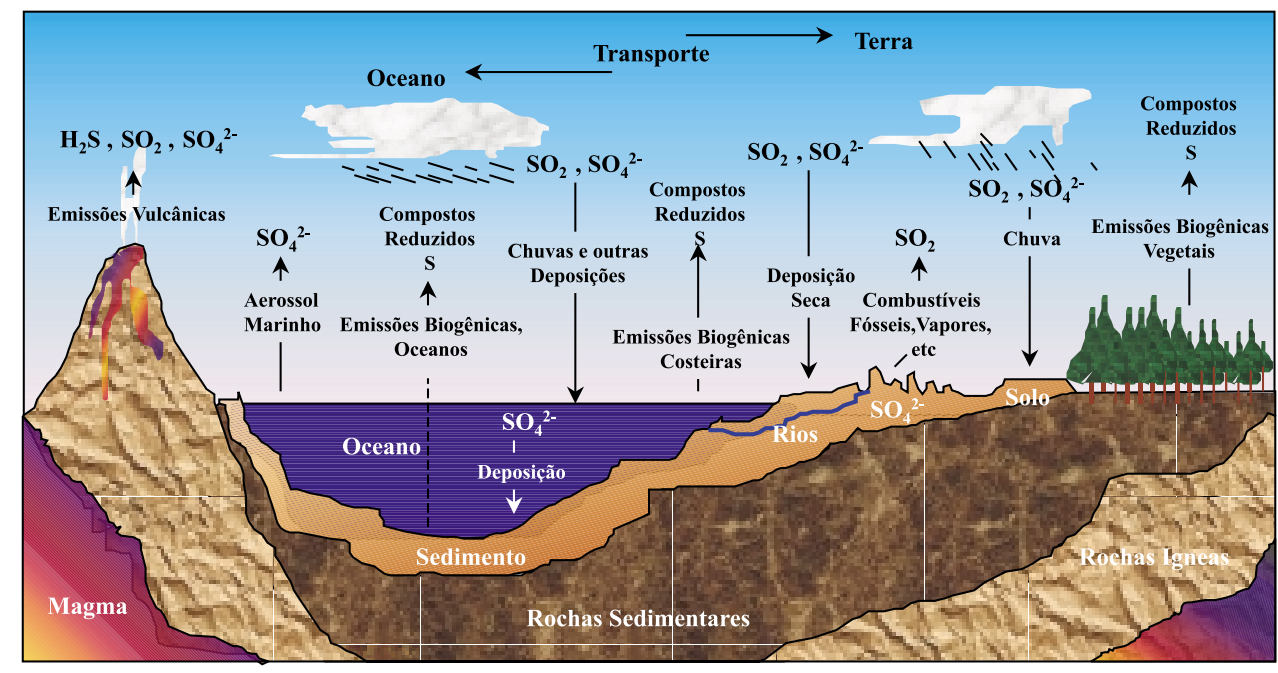

Figura 2. Ciclo do Enxofre (adaptado da Ref. 30) 
e América do $\mathrm{Sul}^{9}$. Nos últimos anos, no entanto, tem sido registrada em muitos países, uma redução na emissão de $\mathrm{SO}_{2}$ e outros poluentes (exceto para os óxidos de nitrogênio), como resultado de programas especiais de despoluição do ar atmosférico ${ }^{9}$.

\section{Óxidos de nitrogênio}

Os óxidos de nitrogênio $\left(\mathrm{NO}_{\mathrm{x}}\right)$ são emitidos, principalmente, sob duas formas: óxido nítrico $(\mathrm{NO})$ e dióxido de nitrogênio $\left(\mathrm{NO}_{2}\right)$. $\mathrm{O}$ óxido nítrico é um importante poluente primário, emitido tanto por fontes móveis quanto por fontes estacionárias. Quantidades menores de $\mathrm{NO}_{2}$ são também emitidas juntamente com $\mathrm{NO}$, sendo que este último pode ser convertido a $\mathrm{NO}_{2}$, no ar atmosférico. Dessa maneira, $\mathrm{NO}_{2}$ é tanto poluente primário quanto poluente secundário, isto é, tanto é emitido diretamente para a atmosfera, como também é formado a partir de reações químicas.

A oxidação do $\mathrm{NO}$ por $\mathrm{O}_{2}$ é muita lenta (eq.1) para ter significado em termos das quantidades presentes na atmosfera ${ }^{2}$. Entretanto, $\mathrm{NO}$ pode reagir com outras espécies tais como $\mathrm{HO}_{2}$ e $\mathrm{RO}_{2}$, em atmosferas limpas ou poluídas, numa seqüência de reações em cadeia, sendo convertido a $\mathrm{NO}_{2}$. Também reage com $\mathrm{O}_{3}$, produzindo $\mathrm{NO}_{2}$, reação esta responsável pela diminuição da concentração de ozônio, em áreas urbanas (eq. 2):

$$
\begin{aligned}
& 2 \mathrm{NO}+\mathrm{O}_{2} \rightarrow 2 \mathrm{NO}_{2} \quad \mathrm{k}^{298 \mathrm{~K}}=2,0 \times 10^{-38} \mathrm{~cm}^{6} \text { molécula }^{-2} \mathrm{~s}^{-1}(1) \\
& \mathrm{NO}+\mathrm{O}_{3} \rightarrow \mathrm{NO}_{2}+\mathrm{O}_{2}
\end{aligned}
$$

Tanto o NO quanto o $\mathrm{NO}_{2}$ são poluentes com papel fundamental na formação do "smog" fotoquímico. As maiores contribuições de $\mathrm{NO}_{2}$ para o ambiente são devidas ao tráfego de automóveis (responsável por cerca de $70 \%$ do $\mathrm{NO}_{\mathrm{x}}$ total emitido) e usinas de energia elétrica que utilizam a queima de combustíveis fósseis. Estima-se que a emissão global de óxidos de nitrogênio corresponda a 50-120 $\mathrm{x} 10^{12} \mathrm{~g}$ de $\mathrm{N}$ ano ${ }^{-1(9)}$. A Tabela 3 apresenta dados de emissão de $\mathrm{NO}_{\mathrm{x}}$ para algumas localidades.

Tabela 3. Emissões Antropogênicas e Naturais de $\mathrm{NO}_{x}\left(\mathrm{~g}\right.$ de $\left.\mathrm{N}^{a n o}{ }^{-1}\right)$

\begin{tabular}{lllc}
\hline Antropogênico & Natural & Local & Ref. \\
\hline $16-48 \times 10^{12}$ & $9-70 \times 10^{12}$ & Escala Global & 38 \\
$13,2-57,4 \times 10^{12}$ & $5-23 \times 10^{12}$ & Hemisfério Norte & 39 \\
$6,5-19 \times 10^{12}$ & $1,5-9,4 \times 10^{12}$ & Hemisfério Sul & 39 \\
$19,8 \times 10^{12}$ & & EUA(1988) & 40 \\
$6,7 \times 10^{12}$ & & RU(1990) & 41 \\
$2,4 \times 10^{12}$ & & América do Sul(1980) & 42 \\
\hline
\end{tabular}

Medidas das concentrações de $\mathrm{NO}$ e $\mathrm{NO}_{2}$ em ambientes urbanos (EUA, 1972) indicaram valores médios horários, de 24 ppb para NO, e de $37 \mathrm{ppb}$ para $\mathrm{NO}_{2}{ }_{2}^{43}$. Em atmosferas poluídas, picos de $\mathrm{NO}_{2}$ de até $800 \mathrm{ppb}$, já foram observados em zonas urbanas, tais como os registrados na área de Los Angeles ${ }^{2}$. No Brasil, o monitoramento deste poluente na zona de Cubatão, São Paulo, durante a década de 80 , indicou concentrações médias diárias de $64 \mu \mathrm{g} \mathrm{m}^{-3}$, sendo que os valores mais altos foram encontrados em estações próximas às rodovias, bem como nas áreas de influência direta da refinaria de petróleo ${ }^{36}$. Medidas realizadas em Salvador, BA, na década de 90, revelaram concentrações de $\mathrm{NO}_{\mathrm{x}}$ na faixa de 11,1 a $36,2 \mathrm{ppb}^{44}$.

\section{Metais}

Compostos gasosos e material particulado são emitidos, a partir de fontes naturais e antropogênicas. Estima-se que a fração de aerossóis emitida a partir de fontes antropogênicas corresponda a aproximadamente $10 \%$ da poluição antropogênica total no $\operatorname{ar}^{9}$. Os principais constituintes de aerossóis naturais são as partículas de aerossol marinho, enquanto que as fontes antropogênicas correspondem principalmente às emissões veiculares, indústria metalúrgica e de construção. Aerossóis emitidos de fontes antropogênicas são formados em maior parte pelos óxidos metáli$\cos$. A quantidade de metais emitida para a atmosfera torna-se um parâmetro importante, desde que muitos dos processos de oxidação atmosféricos podem ser influenciados por íons metálicos.

A emissão antropogênica de metais exibiu um drástico aumento desde o início do século. Por exemplo, a quantidade de $\mathrm{Cu}, \mathrm{Pb}$ e Ni globalmente emitida, aumentou de 1901 a 1980, respectivamente, em $1103 \%, 905 \%$ e $5060 \%{ }^{45}$. Nos últimos anos, porém, em alguns países, a quantidade total de chumbo emitida decresceu em $28 \%$, no período 1981-1990, como conseqüência da substituição de aditivos contendo $\mathrm{Pb}$, pelo MMT (metilciclopentadieniltricarbonilmanganês $)^{46}$. Por sua vez, a quantidade de Mn emitida aumentou em $10 \%$ durante o mesmo período.

Vários estudos têm sido realizados em todo o mundo e gerado uma grande quantidade de informações com relação à presença de metais nos aerossóis atmosféricos ${ }^{47-49}$. No entanto, existe pouca informação disponível sobre o ar no Brasil. Em 1996, de Andrade e colaboradores ${ }^{50}$ determinaram concentrações para diversos metais presentes no aerossol atmosférico, na cidade de Salvador, Brasil. Na Tabela 4 estão colocados alguns dados referentes à coleta realizada em dois bairros da cidade de Salvador. Neste estudo, pode ser observado que $50 \%$ do material particulado foi proveniente de fontes naturais (solo e aerossol marinho), enquanto que $50 \%$ teve origem nas emissões veiculares, indústria metalúrgica e de construção. Medidas de concentrações de metais feitas na cidade do Rio de Janeiro, Brasil (agosto de 1975 a abril de 1978), indicaram valores altos de V, Ni, $\mathrm{Fe}, \mathrm{Mn}, \mathrm{Cr}$ e Zn na área industrial de São Cristovão $0^{51}$. A identificação das fontes destes poluentes correlacionou $\mathrm{V}$ e $\mathrm{Ni}$ à queima de combustíveis em caldeiras industriais; $\mathrm{Fe}, \mathrm{Mn}, \mathrm{Cr}, \mathrm{Cd}, \mathrm{Cu}$ e $\mathrm{Zn}$ como provenientes de indústrias metalúrgicas e processos de chapeamento e galvanização.

Tabela 4. Concentração média de metais na atmosfera da cidade de Salvador ${ }^{50}$

\begin{tabular}{ccc}
\hline & \multicolumn{2}{c}{ Concentração $\mu \mathrm{g} \mathrm{m}^{-3}$} \\
\cline { 2 - 3 } Elemento & Canela & Bonfim \\
\hline $\mathrm{Cd}$ & 0,34 & 0,030 \\
$\mathrm{Co}$ & 0,50 & 0,028 \\
$\mathrm{Cr}$ & 0,16 & 0,16 \\
$\mathrm{Cu}$ & $<0,05$ & 1,1 \\
$\mathrm{Fe}$ & 4,5 & 2,6 \\
$\mathrm{Mn}$ & 0,89 & 0,035 \\
$\mathrm{~Pb}$ & 0,95 & 1,0 \\
$\mathrm{Zn}$ & 0,22 & 0,16 \\
\hline
\end{tabular}

Com relação à concentração dos metais na fase atmosférica aquosa, esta pode variar significativamente, a depender não só da área considerada, como também do fato da medida estar sendo realizada em água de chuva, nuvens ou neblina. Na Tabela 5 estão colocados valores relativos às faixas de concentrações para alguns metais provenientes de medidas em várias localidades. Como pode ser visto, faixas de concentrações mais altas são verificadas em água de neblina. A concentração de Fe determinada em neblina em Bakersfield (EUA), janeiro de 1982, chegou a $6600 \mu \mathrm{g} \mathrm{L}^{-1}$. Já a concentração proveniente da análise em nuvens, na cidade de Los Angeles, em 
novembro de 1981, ficou na faixa de $14-1260 \mu \mathrm{g} \mathrm{L}^{-1}$, enquanto que valores de 1,6-13,2 $\mu \mathrm{g} \mathrm{L}^{-1}$ foram encontrados em água de chuva nos anos de 1981-1982 na mesma cidade ${ }^{14}$.

Tabela 5. Concentração de metais em fase aquosa atmosférica ${ }^{12,14}$

\begin{tabular}{lll}
\hline $\mathrm{Fe}\left(\mu \mathrm{g} \mathrm{L}^{-1}\right)$ & $\mathrm{Mn}\left(\mu \mathrm{g} \mathrm{L}^{-1}\right)$ & Local \\
\hline $240-6600$ & - & neblina (Bakeersfield) \\
$1020-2080$ & $25-81$ & neblina (Lenox) \\
$14-1260$ & $1,5-59$ & nuvem (Los Angeles) \\
$1,6-13,2$ & $1-2,8$ & chuva (Los Angeles) \\
$50-200$ & $100-400$ & nuvem (Ljubljana) \\
\hline
\end{tabular}

\section{Ozônio}

Oxidantes fotoquímicos são formados na atmosfera, a partir de reações fotoquímicas com o dióxido de nitrogênio e compostos orgânicos voláteis $\left(\mathrm{COV}_{\mathrm{s}}\right)$, sendo o ozônio $\left(\mathrm{O}_{3}\right)$, um dos principais produtos formados a partir deste processo ${ }^{2}$.

Ozônio, uma forma alotrópica do oxigênio, é um gás perceptivelmente azul, cujo odor pode ser sentido em concentrações de até $0,01 \mathrm{ppm}$. É um agente oxidante muito mais poderoso que o $\mathrm{O}_{2}$, reagindo com muitas substâncias sob condições em que o $\mathrm{O}_{2}$ é inerte. Em solução ácida, só perde em poder oxidante para o $\mathrm{F}_{2}$, oxigênio atômico e algumas espécies como radicais $\mathrm{OH}$.

A ação da luz ultravioleta sobre o oxigênio produz ozônio na atmosfera superior, estratosfera, sendo que a concentração máxima ocorre numa altura de cerca de $25 \mathrm{Km}$. O ozônio formado na estratosfera é de vital importância para a proteção da superfície terrestre contra a exposição de luz ultravioleta. No entanto, o ozônio formado na troposfera, em contraste com aquele formado em camadas mais altas, constitui um poluente secundário, sendo nocivo para a vida em geral (seres humanos, animais, plantas) e diversos materiais tais como borrachas e tecidos ${ }^{2}$.

A formação do ozônio na troposfera ocorre através da fotólise do $\mathrm{NO}_{2}$, a qual resulta na liberação do oxigênio atômico. Este, por sua vez, se combina com o oxigênio molecular, produzindo ozônio. $\mathrm{Na}$ ausência de outras espécies oxidantes, o NO será oxidado pelo ozônio a $\mathrm{NO}_{2}$, resultando um estado de equilíbrio no qual a concentração de ozônio deve se manter constante:

$\mathrm{NO}_{2}+\mathrm{hv} \rightarrow \mathrm{NO}+\mathrm{O}$

$\mathrm{O}_{2}+\mathrm{O}+\mathrm{M} \rightarrow \mathrm{O}_{3}+\mathrm{M}$

$\mathrm{NO}+\mathrm{O}_{3} \rightarrow \mathrm{NO}_{2}+\mathrm{O}_{2}$

No entanto, tem se tornado cada vez mais comum a presença de hidrocarbonetos, seus derivados e outros $\mathrm{COV}_{\mathrm{s}}$, na atmosfera, os quais reagem produzindo radicais livres, que por sua vez, oxidam o NO sem destruir o ozônio ${ }^{52}$. Deste modo, a concentração de ozônio na baixa atmosfera, tem atingido freqüentemente níveis elevados. As maiores fontes para a produção de ozônio na baixa atmosfera são o tráfego de automóveis, a queima de combustíveis fósseis e processos industriais que produzem dióxido de nitrogênio. Devido à natureza da sua formação, o ozônio é um poluente difícil de ser controlado, sendo um dos que mais comumente excede as concentrações permitidas em áreas urbanas. O desenvolvimento do "smog" fotoquímico, no qual um grande número de reações acontecem simultaneamente, está geralmente associado à ocorrência de picos de ozônio nas grandes cidades. Os reagentes originais em um episódio de "smog" fotoquímico são o óxido de nitrogênio (NO) e os compostos orgânicos voláteis, além da luz solar, ingrediente vital para a produção de radicais livres. Os produtos finais de um "smog" são o ozônio, o ácido nítrico e espécies orgânicas:

$\mathrm{COV}_{\mathrm{s}}+\mathrm{NO}+$ luz solar $\rightarrow \mathrm{O}_{3}, \mathrm{HNO}_{3}$, orgânicos

Estima-se que, no início do século, a concentração de ozônio no continente europeu fosse da ordem de 2 a $10 \mathrm{ppb}^{53}$. Atualmente concentrações atmosféricas na faixa de 50-100 $\mathrm{ppb}^{2}$ são usualmente encontradas em várias localidades. A maior parte dos países tem estabelecido padrões de concentrações máximas de ozônio em torno de 100 ppb. Por exemplo, nos Estados Unidos o padrão é de 120 ppb e no Canadá, 82 ppb. Em ambientes urbanos, durante a formação do "smog" fotoquímico, a concentração de ozônio ultrapassa em muito os padrões estabelecidos. Nas cidades de Los Angeles, México e Atenas já foram registrados níveis de até $600 \mathrm{ppb}^{52,54}$. No Brasil, o padrão nacional de qualidade do ar é fixado em $160 \mu \mathrm{g} \mathrm{m}^{-3}$ para ozônio $^{55}$. Dados de concentração de ozônio em vários bairros de São Paulo mostraram que este padrão é rotineiramente excedido, atingindo níveis de $500 \mu \mathrm{g} \mathrm{m}^{-3(56)}$.

\section{QUÍMICA EM FASE AQUOSA}

\section{Dióxido de enxofre}

$\mathrm{O}$ dióxido de enxofre, $\mathrm{SO}_{2}$, exibe um boa solubilidade em água, em comparação com outros gases presentes na atmosfera, o que resulta em um enriquecimento de S(IV) na água de chuva, nuvens e neblina (Tabela 6).

Tabela 6. Solubilidade de alguns gases em água ( Pgás = 1 atm )

\begin{tabular}{lcccc}
\hline & \multicolumn{3}{c}{ Solubilidade em água $\left(\mathrm{g} \mathrm{L}^{-1}\right)$} & \\
\cline { 2 - 4 } Gás & $10{ }^{\circ} \mathrm{C}$ & $20{ }^{\circ} \mathrm{C}$ & $25{ }^{\circ} \mathrm{C}$ & Ref. \\
\hline $\mathrm{O}_{2}$ & 0,0545 & 0,0443 & 0,0406 & 57 \\
$\mathrm{O}_{3}$ & 0,802 & 0,610 & & 58 \\
$\mathrm{SO}_{2}$ & 153,9 & 106,6 & 94,1 & 59,60 \\
$\mathrm{NO}$ & 0,0770 & 0,0630 & 0,0577 & 61 \\
$\mathrm{CO}_{2}$ & & 1,69 & 1,45 & 60 \\
\hline
\end{tabular}

A solubilidade do $\mathrm{SO}_{2}$ decresce com a diminuição do $\mathrm{pH}$. Deste modo, diferentes espécies de S(IV) irão predominar na solução aquosa: $\mathrm{SO}_{2} .2 \mathrm{H}_{2} \mathrm{O}(\mathrm{pH}<1,5), \mathrm{HSO}_{3}{ }^{-}(\mathrm{pH} 1,5-6,5)$ e $\mathrm{SO}_{3}{ }^{2-}(\mathrm{pH}>6,5), \mathrm{o}$ que pode ser verificado pelas equações que seguem 9 .

$$
\begin{array}{ll}
\mathrm{SO}_{2}(\mathrm{~g})+\mathrm{H}_{2} \mathrm{O}=\mathrm{SO}_{2} \cdot \mathrm{H}_{2} \mathrm{O} & \mathrm{K}=1,23 \mathrm{~mol} \mathrm{~L}^{-1} \mathrm{~atm}^{-1}\left(\mathrm{~T}=25^{\circ} \mathrm{C}\right) \\
\mathrm{SO}_{2} \cdot \mathrm{H}_{2} \mathrm{O}=\mathrm{HSO}_{3}{ }^{-}+\mathrm{H}^{+} & \mathrm{K}=1,32 \times 10^{-2} \mathrm{~mol} \mathrm{~L}^{-1}\left(\mathrm{~T}=25^{\circ} \mathrm{C}\right)(8 \\
\mathrm{HSO}_{3}{ }^{-}=\mathrm{SO}_{3}{ }^{2-}+\mathrm{H}^{+} & \mathrm{K}=6,42 \times 10^{-8} \mathrm{~mol} \mathrm{~L}^{-1}\left(\mathrm{~T}=25^{\circ} \mathrm{C}\right)(9
\end{array}
$$

Assim, na faixa de $\mathrm{pH}$ correspondente à água atmosférica $(\mathrm{pH} 2$ a 6), a maior parte do $\mathrm{SO}_{2}$ dissolvido encontra-se na forma do íon bissulfito, $\mathrm{HSO}_{3}^{-}$. Pode-se observar também, a partir das equações 7 a 9, que em gotas mais ácidas o equilíbrio estará mais deslocado para a esquerda, e portanto menos $\mathrm{SO}_{2}$ ficará dissolvido. A Figura 3 mostra concentrações de $\mathrm{S}(\mathrm{IV})$ em solução aquosa, em equilíbrio com $\mathrm{SO}_{2}$ na fase gasosa, para concentrações de 0,2 a 200 ppb de $\mathrm{SO}_{2}$ e pH da solução de 0 a $6^{2}$. 


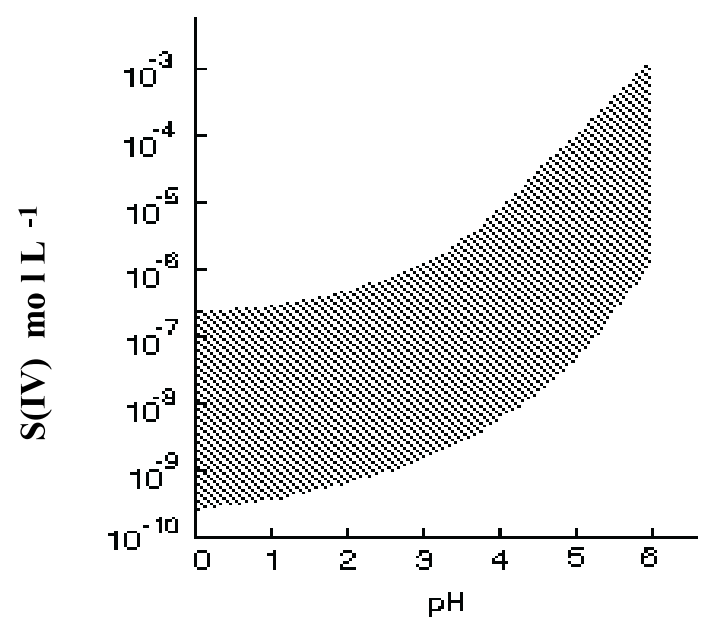

Figura 3. Concentrações de $\mathrm{S}(\mathrm{IV})$ em solução aquosa para $\mathrm{SO}_{2}$ de 0,2 a $200 \mathrm{ppb}^{2}$

$\mathrm{O}$ íon bissulfito, $\mathrm{HSO}_{3}^{-}$, existe em solução aquosa, em duas formas tautoméricas, I e II

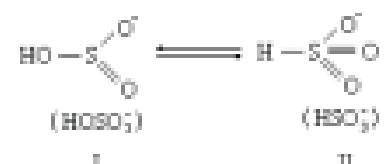

A forma I está presente em solução aquosa, somente em concentrações muito baixas, quando comparada à forma $\mathrm{II}^{62,63}$. A ligação $\mathrm{H}-\mathrm{S}$ parece ser energeticamente mais favorável ${ }^{64}$. A depender da concentração, pode ocorrer a dimerização e o dímero formado estará em equilíbrio com o anion $\mathrm{S}_{2} \mathrm{O}_{5}{ }^{2-(65)}$.
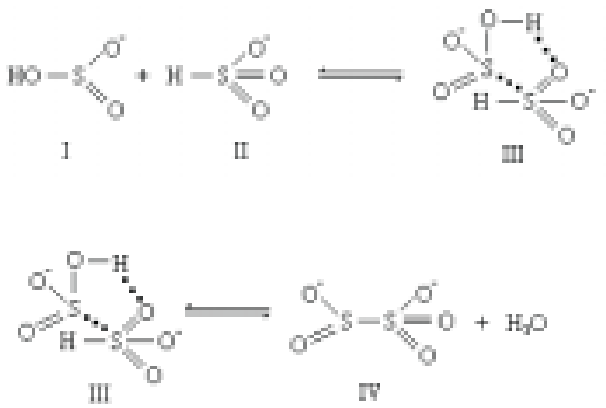

A reatividade química do íon bissulfito é melhor explicada em termos da forma tautomérica I, e pelo rápido equilíbrio estabelecido entre as duas formas. A estereoquímica piramidal da forma I a torna mais suscetível a um ataque nucleofílico do que a forma tetraédrica II ${ }^{9}$.

\section{Metais de transição}

A hidrólise de íons metálicos de transição pode ser representada genericamente pela equação 13 , onde $\mathrm{M}^{\mathrm{z}+}$ representa o cátion metálico e $\mathrm{A}^{\mathrm{n}-} \mathrm{o}$ anion em solução:

$\mathrm{M}^{\mathrm{z}+}+\mathrm{yH}_{2} \mathrm{O}+\mathrm{a} \mathrm{A}^{\mathrm{n}-}=\left[\mathrm{M}\left(\mathrm{H}_{2} \mathrm{O}\right)_{\mathrm{y}-\mathrm{v}}(\mathrm{OH})_{\mathrm{v}} \mathrm{A}_{\mathrm{a}}\right]^{(\mathrm{z}-\mathrm{v}-\mathrm{na})+}+\mathrm{vH}^{+}$

podendo ser simplificada (eq. 14) quando os anions não são complexantes:

$\mathrm{M}^{\mathrm{z+}}+\mathrm{yH}_{2} \mathrm{O}=\left[\mathrm{M}\left(\mathrm{H}_{2} \mathrm{O}\right)_{\mathrm{y}-\mathrm{v}}(\mathrm{OH})_{\mathrm{v}}\right]^{(\mathrm{z}-\mathrm{v})+}+\mathrm{vH}^{+}$
O comportamento de íons metálicos dissolvidos em água pode ser descrito como função da carga, do raio iônico e da estrutura eletrônica. O complexo aquo-hidroxo-metal pode dimerizar ou polimerizar, dependendo do $\mathrm{pH}$ e da concentração, e as espécies resultantes podem conter pontes do tipo $\mu$-oxo ou $\mu$-hidroxo (eq. 15 e 16):

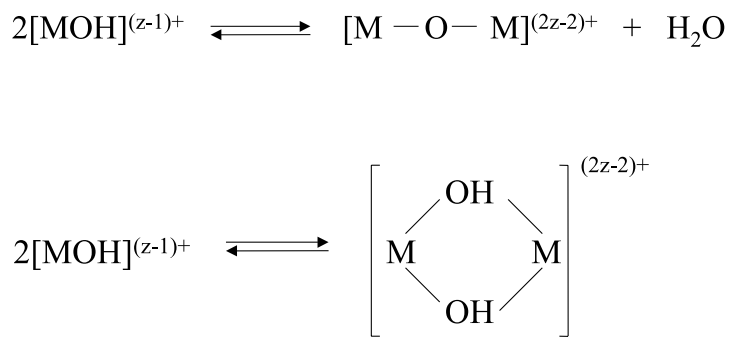

Ferro e manganês são os metais presentes em maior quantidade na atmosfera, apresentando uma química complexa em fase aquosa. Na Tabela 7 podem ser vistas contantes de equilíbrio, relativas à formação de alguns complexos de $\mathrm{Fe}(\mathrm{III})$ e Mn(II) em solução aquo$\mathrm{sa}^{9}$. O íon $\mathrm{Mn}$ (II) não é hidrolisado apreciavelmente, porém em se tratando de $\mathrm{Fe}(\mathrm{III})$, se as soluções não forem fortemente ácidas, ocorre a hidrólise com formação de espécies hidroxo. A partir das constantes de equilíbrio para as espécies formadas, verifica-se que mesmo em $\mathrm{pH}$ da ordem de 2 ou 3, a hidrólise é extensa. Em pH menor do que 1, cerca de $99 \%$ do $\mathrm{Fe}(\mathrm{III})$ em solução está sob a forma de $\left[\mathrm{Fe}\left(\mathrm{H}_{2} \mathrm{O}\right)_{6}\right]^{3+}$. Dependendo do $\mathrm{pH}$ e da concentração, $\mathrm{Fe}(\mathrm{III})$ poderá existir sob diferentes formas, em solução aquosa.

Os complexos de Fe(III) exibem estabilidades e reatividades diferentes. $\mathrm{O}$ íon pentaquohidroxoferro(III) é cerca de 1000 vezes mais reativo que o hexaquoferro(III), provavelmente devido ao efeito de translabilização do ligante hidroxo ${ }^{66,67}$. Outros anions, quando presentes, também podem afetar a estabilidade dos complexos formados. O íon sulfato atua de modo labilizante na estabilidade de soluções de $\mathrm{Fe}(\mathrm{III})$, provocando a formação de espécies diméricas $(\mathrm{FeOH})_{2}{ }^{4+}$ mais rapidamente ${ }^{68}$. Já o íon cloreto estabiliza as espécies poliméricas formadas, pois estes são incorporados na estrutura ${ }^{69}$. A formação de dímeros e polímeros a partir de aquohidroxocomplexos de $\mathrm{Fe}$ (III) é um fator importante a ser considerado, pois diminui a concentração de espécies metálicas cataliticamente ativas.

Complexos aquo-hidroxo-metal reagem rapidamente com $\mathrm{SO}_{2}(\mathrm{aq})$, formando sulfito-complexos, através de ligações $\mathrm{M}-\mathrm{O}$ (3 < $\mathrm{pH}<8$ ). A estabilidade dos complexos metal-sulfito, na atmosfera, é ainda desconhecida. Em águas de nuvem, por exemplo, tem sido constatado que o $\mathrm{Cu}$ se encontra principalmente como $\mathrm{Cu}(\mathrm{I})$; foi então sugerido que o $\mathrm{Cu}$ (II) que é muito mais estável que o $\mathrm{Cu}(\mathrm{I})$, é reduzido pelo sulfito e estabilizado como um complexo de sulfito e $\mathrm{Cu}(\mathrm{I})^{70}$. De acordo com Weschler e $\mathrm{col}^{71}, \mathrm{Cu}$ e Fe existem parcialmente na atmosfera como sulfito-complexos, enquanto que $\mathrm{Mn}$ e $\mathrm{Ni}$ devem existir somente como aquo-complexos ${ }^{72}$.

\section{Oxidantes}

O processo de oxidação do S(IV), por gases dissolvidos na fase aquosa, é possível para diversas espécies, sendo a reação induzida pelo oxigênio a mais investigada. A solubilidade de um gás em água pura pode ser descrita pela lei de Henry (eq. 17).

$\mathrm{c}=\boldsymbol{H} \mathrm{p}_{\mathrm{g}}=\boldsymbol{H} \mathrm{y}_{\mathrm{g}} \mathrm{P}$

onde $\mathrm{c}=$ concentração do gás na solução $\left(\mathrm{mol} \mathrm{L}^{-1}\right)$

$\boldsymbol{H}=$ constante de Henry $\left(\mathrm{mol} \mathrm{L}^{-1} \mathrm{~atm}^{-1}\right)$

$\mathrm{p}_{\mathrm{g}}=$ pressão parcial do gás (atm) 
Tabela 7. Constantes de equilíbrio para alguns complexos de Fe(III) e Mn(II), em solução aquosa9

\begin{tabular}{|c|c|}
\hline Complexos de Fe(III) & $\mathrm{K}$ \\
\hline $\begin{array}{l}{\left[\mathrm{Fe}\left(\mathrm{H}_{2} \mathrm{O}\right)_{6}\right]^{3+}=\left[\mathrm{Fe}\left(\mathrm{H}_{2} \mathrm{O}\right)_{5}(\mathrm{OH})\right]^{2+}+\mathrm{H}^{+}} \\
{\left[\mathrm{Fe}\left(\mathrm{H}_{2} \mathrm{O}\right)_{6}\right]^{3+}=\left[\mathrm{Fe}\left(\mathrm{H}_{2} \mathrm{O}\right)_{4}(\mathrm{OH})_{2}\right]^{+}+2 \mathrm{H}^{+}} \\
{\left[\mathrm{Fe}\left(\mathrm{H}_{2} \mathrm{O}\right)_{5}(\mathrm{OH})\right]^{2+}=\left[\mathrm{Fe}\left(\mathrm{H}_{2} \mathrm{O}\right)_{4}(\mathrm{OH})_{2}\right]^{+}+\mathrm{H}^{+}} \\
2\left[\mathrm{Fe}\left(\mathrm{H}_{2} \mathrm{O}\right)_{6}\right]^{3+}=\left[\left(\mathrm{H}_{2} \mathrm{O}\right)_{5} \mathrm{Fe}(\mathrm{OH}) \mathrm{Fe}\left(\mathrm{H}_{2} \mathrm{O}\right)_{5}\right]^{5+}+\mathrm{H}^{+}+\mathrm{H}_{2} \mathrm{O} \\
{\left[\mathrm{Fe}\left(\mathrm{H}_{2} \mathrm{O}\right)_{6}\right]^{3+}+\mathrm{Cl}-=\left[\mathrm{Fe}\left(\mathrm{H}_{2} \mathrm{O}\right)_{5} \mathrm{Cl}\right]^{2+}+\mathrm{H}_{2} \mathrm{O}} \\
{\left[\mathrm{Fe}\left(\mathrm{H}_{2} \mathrm{O}\right)_{6}\right]^{3+}+2 \mathrm{Cl}^{-}=\left[\mathrm{Fe}\left(\mathrm{H}_{2} \mathrm{O}\right)_{4} \mathrm{Cl}_{2}\right]^{+}+2 \mathrm{H}_{2} \mathrm{O}} \\
{\left[\mathrm{Fe}\left(\mathrm{H}_{2} \mathrm{O}\right)_{6}\right]^{3+}+\mathrm{HSO}_{3}^{-}=\left[\mathrm{Fe}\left(\mathrm{H}_{2} \mathrm{O}\right)_{5}\left(\mathrm{HSO}_{3}\right)\right]^{2+}+\mathrm{H}_{2} \mathrm{O}} \\
{\left[\mathrm{Fe}\left(\mathrm{H}_{2} \mathrm{O}\right)_{5}(\mathrm{OH})\right]^{2+}+\mathrm{HSO}_{3}^{-}=\left[\mathrm{Fe}\left(\mathrm{H}_{2} \mathrm{O}\right)_{4}\left(\mathrm{HSO}_{3}\right)(\mathrm{OH})\right]^{+}+\mathrm{H}_{2} \mathrm{O}} \\
{\left[\mathrm{Fe}\left(\mathrm{H}_{2} \mathrm{O}\right)_{6}\right]^{3+}+2 \mathrm{SO}_{4}{ }^{2-}=\left[\mathrm{Fe}\left(\mathrm{H}_{2} \mathrm{O}\right)_{4}\left(\mathrm{SO}_{4}\right)_{2}\right]^{-}+2 \mathrm{H}_{2} \mathrm{O}} \\
{\left[\mathrm{Fe}\left(\mathrm{H}_{2} \mathrm{O}\right)_{6}\right]^{3+}+\mathrm{H}_{3} \mathrm{CCOOH}=\left[\mathrm{Fe}\left(\mathrm{H}_{2} \mathrm{O}\right)_{5}\left(\mathrm{H}_{3} \mathrm{CCOO}\right)\right]^{2+}+\mathrm{H}_{2} \mathrm{O}+\mathrm{H}^{+}}\end{array}$ & $\begin{array}{l}2,3 \times 10^{-3} \mathrm{~mol} / \mathrm{L} \\
5,1 \times 10^{-7}(\mathrm{~mol} / \mathrm{L})^{2} \\
9,8 \times 10^{-4} \mathrm{~mol} / \mathrm{L} \\
1,05 \times 10^{-1} \\
1,5(\mathrm{~mol} / \mathrm{L})^{-1} \\
0,44(\mathrm{~mol} / \mathrm{L})^{-2} \\
55(\mathrm{~mol} / \mathrm{L})^{-1} \\
600(\mathrm{~mol} / \mathrm{L})^{-1} \\
9 \times 10^{2}(\mathrm{~mol} / \mathrm{L})^{-1} \\
1,1 \times 10^{3}\end{array}$ \\
\hline Complexos de $\mathrm{Mn}(\mathrm{II})$ & $\mathrm{K}$ \\
\hline $\begin{array}{l}{\left[\mathrm{Mn}\left(\mathrm{H}_{2} \mathrm{O}\right)_{6}\right]^{2+}=\left[\mathrm{Mn}\left(\mathrm{H}_{2} \mathrm{O}\right)_{5}(\mathrm{OH})\right]^{+}+\mathrm{H}^{+}} \\
{\left[\mathrm{Mn}\left(\mathrm{H}_{2} \mathrm{O}\right)_{6}\right]^{2+}=\left[\mathrm{Mn}\left(\mathrm{H}_{2} \mathrm{O}\right)_{4}(\mathrm{OH})_{2}\right]^{+}+2 \mathrm{H}^{+}} \\
2\left[\mathrm{Mn}\left(\mathrm{H}_{2} \mathrm{O}\right)_{6}\right]^{2+}=\left[\left(\mathrm{H}_{2} \mathrm{O}\right)_{4} \mathrm{Mn}(\mathrm{OH}) \mathrm{Mn}\left(\mathrm{H}_{2} \mathrm{O}\right)_{4}\right]^{3+}+\mathrm{H}^{+}+\mathrm{H}_{2} \mathrm{O} \\
{\left[\mathrm{Mn}\left(\mathrm{H}_{2} \mathrm{O}\right)_{6}\right]^{2+}+2 \mathrm{Cl}^{-}=\left[\mathrm{Mn}\left(\mathrm{H}_{2} \mathrm{O}\right)_{4} \mathrm{Cl}_{2}\right]+2 \mathrm{H}_{2} \mathrm{O}} \\
{\left[\mathrm{Mn}\left(\mathrm{H}_{2} \mathrm{O}\right)_{6}\right]^{2+}+\mathrm{SO}_{4}^{2-}=\left[\mathrm{Mn}\left(\mathrm{H}_{2} \mathrm{O}\right)_{5}\left(\mathrm{SO}_{4}\right)\right]+\mathrm{H}_{2} \mathrm{O}} \\
{\left[\mathrm{Mn}\left(\mathrm{H}_{2} \mathrm{O}\right)_{6}\right]^{2+}+\mathrm{H}_{3} \mathrm{CCOOH}=\left[\mathrm{Mn}\left(\mathrm{H}_{2} \mathrm{O}\right)_{5}\left(\mathrm{H}_{3} \mathrm{CCOO}\right)\right]^{+}+\mathrm{H}_{2} \mathrm{O}+\mathrm{H}^{+}}\end{array}$ & $\begin{array}{l}2,82 \times 10^{-11} \mathrm{~mol} / \mathrm{L} \\
1,09 \times 10^{-13} \mathrm{~mol} / \mathrm{L} \\
1,35 \times 10^{-10} \\
1,80(\mathrm{~mol} / \mathrm{L})^{-2} \\
133(\mathrm{~mol} / \mathrm{L})^{-1} \\
6,3\end{array}$ \\
\hline
\end{tabular}

$$
\begin{aligned}
& \mathrm{y}_{\mathrm{g}}=\text { fração molar do gás } \\
& \mathrm{P}=\text { pressão total acima da solução (atm) }
\end{aligned}
$$

Se outras substâncias estão dissolvidas na solução, a solubilidade do gás diminui e pode não seguir a lei de Henry. Na Tabela 8 estão colocados valores de concentração de $\mathrm{O}_{2}$ dissolvido em água pura e em algumas soluções de sais ${ }^{9}$.

Tabela 8. Solubilidade do $\mathrm{O}_{2}$ em água e em soluções aquosas a $25^{\circ}$ $\mathrm{C}\left(\mathrm{pO}_{2}=1 \mathrm{~atm}\right)^{9}$

\begin{tabular}{ccc}
\hline sal & {$[\mathrm{sal}] \mathrm{mol} \mathrm{L}^{-1}$} & {$\left[\mathrm{O}_{2}\right] \mathrm{mol} \mathrm{L}^{-1}$} \\
\hline & & $1,27 \times 10^{-3}$ \\
$\mathrm{Na}_{2} \mathrm{SO}_{3}$ & 0,1 & $1,15 \times 10^{-3}$ \\
$\mathrm{Na}_{2} \mathrm{SO}_{4}$ & 0,15 & $1,12 \times 10^{-3}$ \\
& 0,50 & $8,13 \times 10^{-4}$ \\
$\mathrm{NaCl}$ & 0,25 & $1,24 \times 10^{-3}$ \\
\hline
\end{tabular}

Pelos valores de concentração de $\mathrm{O}_{2}$ dissolvido, observa-se pequenos desvios em relação à lei de Henry no processo de solubilização deste gás em soluções aquosas diluídas. $\mathrm{O}$ pH da solução tem pouca influência na solubilidade do oxigênio, como pode ser observado pelos valores de concentração de $\mathrm{O}_{2}$ apresentados na Tabela $9^{73}$.

Tabela 9. Solubilidade do oxigênio em solução aquosa de $\mathrm{Na}_{2} \mathrm{SO}_{3}$ $\left(0,8\right.$ molL $\left.^{-1}\right)$ saturada com ar, a $25{ }^{\circ} \mathrm{C}$, em função do $\mathrm{pH}^{73}$

\begin{tabular}{cc}
\hline $\mathrm{pH}$ & {$\left[\mathrm{O}_{2}\right] \mathrm{mol} \mathrm{L}^{-1}$} \\
\hline 9,0 & $1,53 \times 10^{-4}$ \\
8,0 & $1,53 \times 10^{-4}$ \\
7,5 & $1,53 \times 10^{-4}$ \\
6,5 & $1,52 \times 10^{-4}$ \\
\hline
\end{tabular}

A molécula do oxigênio ( $\left(\mathrm{O}_{2} \cdot\right.$, triplete no estado fundamental), é um biradical com dois elétrons desemparelhados e uma ordem de ligação igual a dois. Toda a química do oxigênio é caracterizada por estas propriedades. Sob condições brandas, o oxigênio reage vagarosamente no processo de oxidação, já que a redução da molécula de oxigênio ocorre em etapas (Figura 4). Pode ser verificado, pelos potenciais fornecidos, que a água neutra saturada por $\mathrm{O}_{2}$ é um agente oxidante muito bom. Porém, muitas oxidações que ocorrem pela ação do oxigênio em solução são lentas; as velocidades podem ser muitíssimo aumentadas pela ação catalítica de pequenas quantidades de íons metálicos de transição, provavelmente devida à formação de complexos ${ }^{74}$. Apesar da entidade $\mathrm{O}_{2}$ manter-se intacta, os complexos podem ser descritos como tendo íons, $\mathrm{O}_{2}{ }^{-} \mathrm{e}_{2}{ }^{2-}$, coordenados ao metal, formando um anel triangular ou uma ponte. O oxigênio coordenado se torna mais reativo que o $\mathrm{O}_{2}$ livre e substâncias que não são diretamente oxidadas sob condições não muito enérgicas, podem ser atacadas na presença de complexos metálicos.
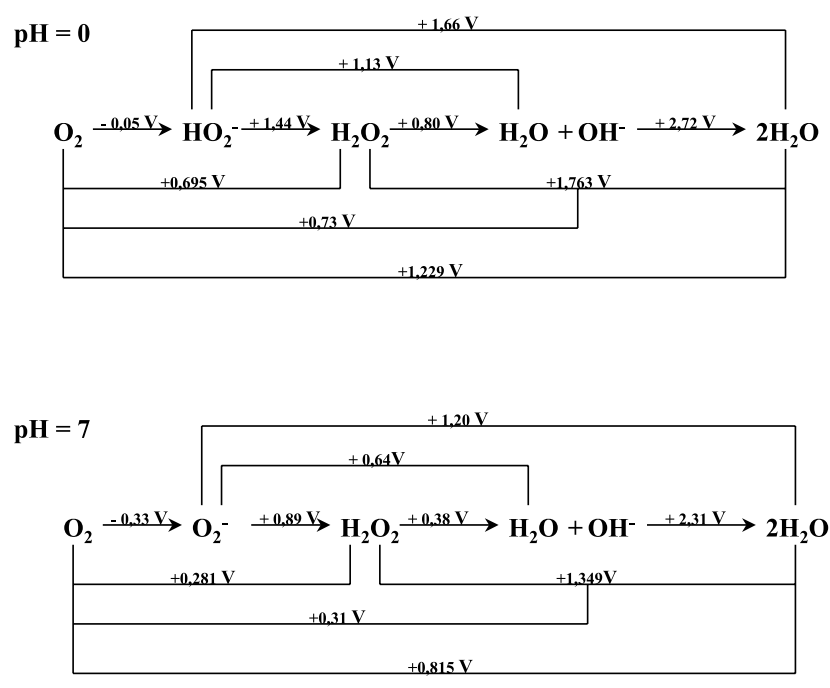

Figura 4. Potenciais de redução do oxigênio em água, $\mathrm{pO}_{2}=1 \mathrm{~atm}^{9}$

Outros oxidantes, tais como $\mathrm{O}_{3}$ e $\mathrm{NO}_{2}$, quando presentes na fase aquosa atmosférica, também podem induzir o processo de oxidação das espécies de $\mathrm{S}(\mathrm{IV})$, via caminhos catalisados, ou não.

O ozônio é um gás solúvel em água (Tabela 6), que pode se decompor neste meio, na presença de $\mathrm{OH}^{-}$, numa sequência de reações, conforme é indicado a seguir": 
$\mathrm{O}_{3}+\mathrm{OH}^{-} \rightarrow \mathrm{O}_{2}^{\cdot-}+\mathrm{HO}_{2}$

$\mathrm{O}_{3}+\mathrm{HO}_{2} \rightarrow \mathrm{OH}^{\cdot}+2 \mathrm{O}_{2}$

$\mathrm{O}_{3}+\mathrm{OH}^{\cdot} \rightarrow \mathrm{HO}_{2}+\mathrm{O}_{2}$

$\mathrm{OH}^{\cdot}+\mathrm{HO}_{2} \rightarrow \mathrm{O}_{2}+\mathrm{H}_{2} \mathrm{O}$

Comparando-se os potenciais do $\mathrm{O}_{2}$ e do $\mathrm{O}_{3}$ em solução aquosa, verifica-se a maior força oxidante do ozônio (eq. 22 e 23):

$\mathrm{O}_{2}+4 \mathrm{H}^{+}\left(10^{-7} \mathrm{~mol} \mathrm{~L}^{-1}\right)+4 \mathrm{e}=2 \mathrm{H}_{2} \mathrm{O} \quad \mathrm{E}^{\mathrm{o}}=+0,815 \mathrm{~V}$

$\mathrm{O}_{3}+2 \mathrm{H}^{+}\left(10^{-7} \mathrm{~mol} \mathrm{~L}^{-1}\right)+2 \mathrm{e}=\mathrm{O}_{2}+\mathrm{H}_{2} \mathrm{O} \quad \mathrm{E}^{\mathrm{o}}=+1,65 \mathrm{~V}$

Reações envolvendo óxidos de nitrogênio como espécies oxidantes e o dióxido de enxofre, são termodinamicamente possíveis e consideradas importantes do ponto de vista ambiental. Dióxido de nitrogênio, $\mathrm{NO}_{2}$, é muito mais solúvel que o $\mathrm{NO}$, e em solução ácida, dimeriza rapidamente estabelecendo um equilíbrio com a espécie $\mathrm{N}_{2} \mathrm{O}_{4}$ (eq.24) ${ }^{14}$ :

$2 \mathrm{NO}_{2(\text { aq) }}=\mathrm{N}_{2} \mathrm{O}_{4 \text { (aq) }} \quad \mathrm{K}_{\mathrm{d}}=7 \times 10^{4} \mathrm{~mol}^{-1} \mathrm{~L}$

O tetróxido de nitrogênio, por sua vez, constitui um agente oxidante razoavelmente forte, com valores de $\mathrm{E}^{\mathrm{o}}$ de $+1,07 \mathrm{~V}$ e $+1,03$ $\mathrm{V}$, respectivamente, para as equações que seguem ${ }^{14}$ :

$\begin{array}{ll}\mathrm{N}_{2} \mathrm{O}_{4}+2 \mathrm{H}^{+}+2 \mathrm{e}=2 \mathrm{HNO}_{2} & \mathrm{E}^{\mathrm{o}}=+1,07 \mathrm{~V} \\ \mathrm{~N}_{2} \mathrm{O}_{4}+4 \mathrm{H}^{+}+4 \mathrm{e}=2 \mathrm{H}_{2} \mathrm{O}+2 \mathrm{NO} & \mathrm{E}^{\mathrm{o}}=+1,03 \mathrm{~V}\end{array}$

Apesar do $\mathrm{NO}_{2}$ apresentar um valor moderado para a constante de Henry, 1 x 10-2 $\mathrm{mol} \mathrm{L}^{-1} \mathrm{~atm}^{-1}$ (Tabela 10), a solubilidade deste gás é aumentada com a formação do dímero, $\mathrm{N}_{2} \mathrm{O}_{4}$, pois a constante para este último está em torno de $1,4 \mathrm{~mol} \mathrm{~L}^{-1} \mathrm{~atm}^{-1}$. Assim, $\mathrm{NO}_{2}$ juntamente com o dímero $\mathrm{N}_{2} \mathrm{O}_{4}$, dissolvem em solução aquosa em muito maior extensão do que o NO, tendo portanto uma importância maior no que diz respeito aos sistemas aquosos.

Tabela 10. Constantes de Henry para alguns gases atmosféricos, em água líquida, a $25^{\circ} \mathrm{C}$

\begin{tabular}{lcl}
\hline $\mathrm{Gas}$ & $\boldsymbol{H}\left(\mathrm{mol} \mathrm{L}^{-1} \mathrm{~atm}^{-1}\right)$ & Ref. \\
\hline $\mathrm{O}_{2}$ & $1,3 \times 10^{-3}$ & 75 \\
$\mathrm{NO}$ & $1,9 \times 10^{-3}$ & 75 \\
$\mathrm{NO}_{2}$ & $1 \times 10^{-2}$ & 76 \\
$\mathrm{O}_{3}$ & $1,3 \times 10^{-2}$ & 77 \\
$\mathrm{SO}_{2}$ & 1,23 & 75 \\
$\mathrm{~N}_{2} \mathrm{O}_{4}$ & 1,4 & 76 \\
\hline
\end{tabular}

\section{Reação de oxidação do S(IV) em presença de oxigênio}

A oxidação do $\mathrm{S}(\mathrm{IV})$ em fase aquosa, por $\mathrm{O}_{2}$, tem sido estudada por vários pesquisadores ${ }^{9-26}$, sendo que os resultados têm sido objeto de considerável controvérsia, em diversos aspectos. Para reações não catalisadas, a dependência com a concentração do oxigênio é discutida na literatura (Tabela 11). Na maior parte dos casos, não se observa dependência, porém alguns autores encontraram uma dependência de primeira ordem em relação ao $\mathrm{O}_{2}$.

$\mathrm{Na}$ Figura 5 a área hachureada representa os valores das constantes de velocidade, em função do $\mathrm{pH}$ do meio, determinados em diversos estudos ${ }^{86}$. A depender do $\mathrm{pH}$, podem ser observadas discre-
Tabela 11. Ordens de reação para oxidação de S(IV) por $\mathrm{O}_{2}$

\begin{tabular}{cll}
\hline ordem em relação ao $\mathrm{O}_{2}$ & condições & Ref. \\
\hline 0 & {$\left[\mathrm{O}_{2}\right]>0,8 \mathrm{mg} \mathrm{L}^{-1}$} & 78 \\
0 & {$\left[\mathrm{O}_{2}\right]: 0,02-0,5 \mathrm{mmol} \mathrm{L}^{-1}$} & 79 \\
0 & $\mathrm{pO}_{2}: 120-700 \mathrm{mmHg}$ & 80 \\
0 & $\mathrm{pO}_{2}: 0,11-1 \mathrm{~atm}$ & 81 \\
0 & {$\left[\mathrm{O}_{2}\right]: 0,26-1,25 \mathrm{mmol} \mathrm{L}^{-1}$} & 82,83 \\
0 & {$\left[\mathrm{O}_{2}\right]: 0,26-0,43 \mathrm{mmol} \mathrm{L}^{-1}$} & 84 \\
1 & $\mathrm{pO}_{2}: 0,02-1 \mathrm{~atm}$ & 85 \\
1 & {$\left[\mathrm{O}_{2}\right]: 0,26-1,25 \mathrm{mmol} \mathrm{L}^{-1}$} & 73 \\
\hline
\end{tabular}

pâncias maiores do que duas ordens de grandeza nas constantes de velocidade, o que reflete toda a dificuldade experimental para o estudo deste tipo de reação. Estas dificuldades parecem incluir: a presença de íons metálicos como impurezas, na água ou nos reagentes, que possam catalisar a oxidação do S(IV); a presença de compostos orgânicos como impurezas, que podem agir como inibidores para a reação; problemas de transporte de massa que também podem ocorrer, principalmente quando os trabalhos são realizados em concentrações mais elevadas. A dependência da reação com o pH deve estar relacionada com os valores de potenciais redox para o $\mathrm{O}_{2}$ (Figura 4) e com a reatividade das diferentes espécies de $\mathrm{S}(\mathrm{IV})$ formadas.

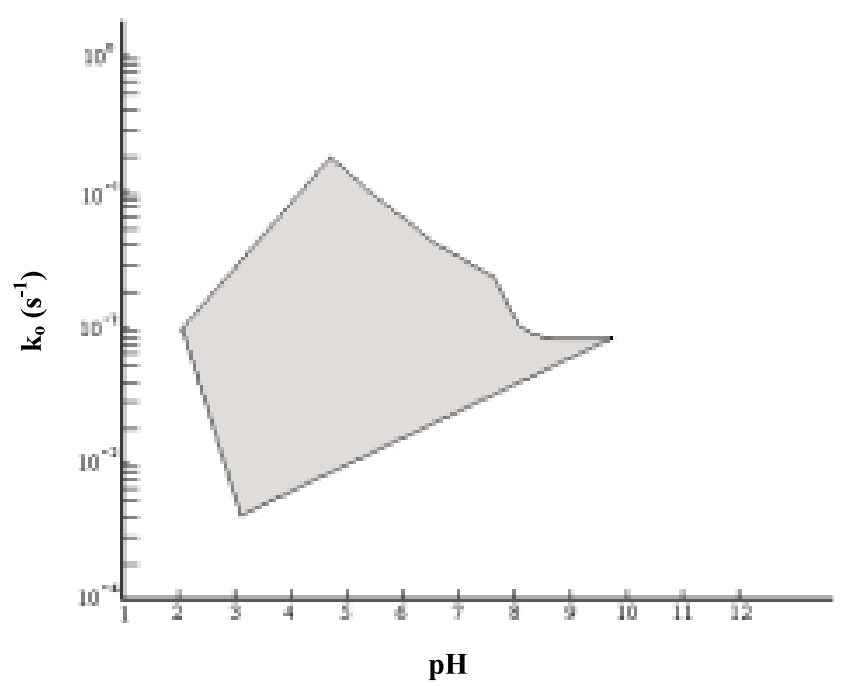

Figura 5. Variação nos valores de constantes cinéticas determinadas por diversos autores, para a reação de oxidação do $S(I V)_{\text {aq }}$ por $\mathrm{O}_{2}$ (adaptado da Ref. 86)

\section{Reação de oxidação do S(IV) em presença de $\mathrm{O}_{2}$ e íons metálicos de transição}

A atividade catalítica de íons metálicos de transição, no processo de oxidação do S(IV), tem sido estudada por diversos pesquisadores, porém os resultados publicados mostram divergências com relação a que metais são catalisadores mais ativos para este tipo de reação. A Tabela 12 sumariza resultados para experimentos envolvendo os íons metálicos mais comumente estudados, conduzidos em vários laboratórios ${ }^{9}$. O fato de se encontrar diferentes resultados, provavelmente se deve às condições em que os experimentos foram conduzidos, tais como: faixa de concentração das espécies participantes, $\mathrm{pH}$ do meio, temperatura, força iônica, presença de impurezas. O estudo da influência de outros íons, tais como $\mathrm{Cr}(\mathrm{VI}), \mathrm{Cr}(\mathrm{III})$, $\mathrm{Cd}(\mathrm{II})$ e $\mathrm{V}(\mathrm{V})$, na oxidação do $\mathrm{S}(\mathrm{IV})$ em fase aquosa, tem sido pouco relatado na literatura ${ }^{87,88}$. Martins e colaboradores ${ }^{87}$ encontraram que $^{-}$ 
a reação de oxidação do $\mathrm{S}(\mathrm{IV})$ teve sua velocidade aumentada na presença de $\mathrm{Cr}(\mathrm{VI})$, na faixa de $\mathrm{pH} 2,1-4,5$, porém nenhum efeito catalítico foi observado para o íon Cd(II) e efeitos catalíticos inibitórios foram observados para os íons $\mathrm{Cr}(\mathrm{III})$ e $\mathrm{V}(\mathrm{V})$.

Tentativas para se entender a habilidade catalítica dos íons metálicos de transição, tem sido feitas em termos da possibilidade de ocorrerem estados de oxidação múltiplos e da energia necessária para que o íon atinja um estado de oxidação mais elevado. Para que um ín seja cataliticamente ativo, este deve ser capaz de ascender a um estado de oxidação suficientemente alto, de maneira que possa extrair um elétron da espécie de S(IV), gerando então, intermediários ativos, que podem propagar a reação. Deve haver, portanto, uma etapa energeticamente favorável, na qual o íon metálico reduzido retorne a um estado de oxidação mais elevado. Este tem sido um aspecto importante e bastante discutido : como a espécie metálica reduzida seria reoxidada em seguida, completando o ciclo catalítico.

O mecanismo através do qual ocorre a catálise homogênea por metal de transição, também tem sido matéria de discussão na literatura $^{2,14,24}$. Nesse sentido, três tipos de mecanismos tem sido propostos: 1) por radical; 2) não envolvendo radical; 3) combinação dos dois mecanismos anteriores. A maior parte das publicações sobre o assunto explica o processo através do mecanismo por radical livre, que foi proposto pela primeira vez por Bäckstrom ${ }^{92}$ conforme a seqüência de reações:

$$
\begin{aligned}
& \text { Iniciação } \quad \mathrm{M}^{\mathrm{n}+}+\mathrm{SO}_{3}^{2-} \rightarrow \mathrm{M}^{(\mathrm{n}-1)+}+\mathrm{SO}_{3}{ }^{\cdot-} \\
& \text { Propagação } \mathrm{SO}_{3}^{\cdot-}+\mathrm{O}_{2} \rightarrow \mathrm{SO}_{5}{ }^{\cdot-} \\
& \mathrm{SO}_{5} \cdot{ }^{--}+\mathrm{SO}_{3}^{2-} \rightarrow \mathrm{SO}_{5}^{2-}+\mathrm{SO}_{3}{ }^{\cdot} \\
& \text { Oxidação } \quad \mathrm{SO}_{5}{ }^{2-}+\mathrm{SO}_{3}{ }^{2-} \rightarrow 2 \mathrm{SO}_{4}{ }^{2-} \\
& \text { Término } 2 \mathrm{SO}_{3}{ }^{--} \rightarrow 2 \mathrm{~S}_{2} \mathrm{O}_{6}{ }^{2-} \\
& \mathrm{SO}_{3}{ }^{--}+\mathrm{SO}_{5}^{\cdot-} \rightarrow 2 \mathrm{~S}_{2} \mathrm{O}_{6}{ }^{2-}+\mathrm{O}_{2} \\
& \mathrm{SO}_{5} \cdot{ }^{--}+\mathrm{SO}_{5} \cdot{ }^{\cdot-} \rightarrow 2 \mathrm{~S}_{2} \mathrm{O}_{8}^{2-}+\mathrm{O}_{2}
\end{aligned}
$$

Desde então, muitos autores têm utilizado mecanismos similares para justificar seus resultados experimentais. No entanto, em uma série de trabalhos, este mecanismo se mostrou inconsistente para explicar dados obtidos, tais como, a ordem de reação em relação a cada espécie envolvida. Mecanismos alternativos foram então desenvolvidos.

O mecanismo polar (não envolvendo formação de radicais), foi proposto por Basset e Parker ${ }^{93}$, para explicar o efeito catalítico dos íons $\mathrm{Mn}^{2+}$ na autoxidação do S(IV). Este mecanismo apresenta uma etapa inicial, onde há a formação de um complexo de esfera interna metal-sulfito. Em seguida ocorre a reação entre o oxigênio e o complexo metal-sulfito, formando um complexo oxigenado, e finalmente a transferência de dois elétrons (eq.34 a 37):

$\mathrm{Mn}^{2+}+\mathrm{SO}_{3}{ }^{2-}=\mathrm{MnSO}_{3}{ }^{0}$

$\mathrm{MnSO}_{3}{ }^{0}+\mathrm{SO}_{3}{ }^{2-}=\mathrm{Mn}\left(\mathrm{SO}_{3}\right)_{2}^{2-}$

$\mathrm{Mn}\left(\mathrm{SO}_{3}\right)_{2}^{2-}+\mathrm{O}_{2}=\mathrm{Mn}\left(\mathrm{SO}_{3}\right)_{2} \mathrm{O}_{2}^{2-}$

$\mathrm{Mn}\left(\mathrm{SO}_{3}\right)_{2} \mathrm{O}_{2}^{2-} \rightarrow \mathrm{Mn}^{2+}+2 \mathrm{SO}_{4}^{2-}$

Outros autores $^{21,94}$ também utilizaram mecanismos similares para explicar a autoxidação catalítica de S(IV), por Fe(III) e $\mathrm{Cu}(\mathrm{I})$.

A maior parte dos trabalhos desenvolvidos tem sido justificada, no entanto, pelo mecanismo via formação de radical, e alguns autores sugerem que o íon metálico reduzido, poderia ser reoxidado pelos próprios radicais formados no processo. No caso do $\mathrm{Fe}(\mathrm{II}) / \mathrm{Fe}(\mathrm{III})$, um dos sistemas com maior atividade catalítica, Brandt e colaboradores $^{24}$, propõem o seguinte esquema de reações em um mecanismo por radicais em cadeia:

$$
\begin{aligned}
& \mathrm{Fe}(\mathrm{III})+\mathrm{nHSO}_{3}^{-}=\mathrm{Fe}^{\mathrm{III}}\left(\mathrm{HSO}_{3}^{-}\right)_{\mathrm{n}}^{3-\mathrm{n}} \quad(\mathrm{n}=1,2,3) \\
& \text { Redução } \mathrm{Fe}^{\mathrm{III}}\left(\mathrm{HSO}_{3}{ }^{-}\right)_{\mathrm{n}}{ }^{3-\mathrm{n}} \rightarrow \mathrm{Fe}(\mathrm{II})+(\mathrm{n}-1) \mathrm{HSO}_{3}^{-}+\mathrm{H}^{+}+\mathrm{SO}_{3}^{-} \\
& \mathrm{Fe}(\mathrm{III})+\mathrm{SO}_{3}{ }^{-}+\mathrm{H}_{2} \mathrm{O} \rightarrow \mathrm{Fe}(\mathrm{II})+\mathrm{HSO}_{4}{ }^{-}+\mathrm{H}^{+} \\
& \mathrm{SO}_{3} \cdot{ }^{-}+\mathrm{O}_{2} \rightarrow \mathrm{SO}_{5} \cdot \\
& \mathrm{SO}_{5}^{\cdot-}+\mathrm{HSO}_{3}^{-} \rightarrow \mathrm{SO}_{3} \cdot{ }^{-}+\mathrm{HSO}_{5} \\
& \mathrm{Fe}(\mathrm{II})+\mathrm{SO}_{5}^{\cdot-}+\mathrm{H}^{+} \rightarrow \mathrm{Fe}(\mathrm{III})+\mathrm{HSO}_{5}{ }^{-} \\
& \text {Oxidação } \mathrm{Fe}(\mathrm{II})+\mathrm{HSO}_{5}{ }^{-} \rightarrow \mathrm{Fe}(\mathrm{III})+\mathrm{SO}_{4}{ }^{-}+\mathrm{OH}^{-} \\
& \mathrm{Fe}(\mathrm{II})+\mathrm{SO}_{4}^{\cdot-}+\mathrm{H}^{+} \rightarrow \mathrm{Fe}(\mathrm{III})+\mathrm{HSO}_{4}^{-} \\
& \mathrm{SO}_{4}^{-{ }^{-}+}+\mathrm{HSO}_{3}^{-} \rightarrow \mathrm{SO}_{3}^{\cdot-}+\mathrm{HSO}_{4}^{-}
\end{aligned}
$$

As primeiras reações representam a redução dos complexos sulfito-Fe(III). Em sequiência, o radical $\mathrm{SO}_{3}{ }^{-}$pode tanto reduzir a espécie $\mathrm{Fe}(\mathrm{III})$, como também formar rapidamente o radical $\mathrm{SO}_{5}{ }^{\cdot-}$, na presença de oxigênio. $\mathrm{O}$ radical $\mathrm{SO}_{5}{ }^{-}$é um agente oxidante forte $\left(\mathrm{E}^{\mathrm{o}} \mathrm{SO}_{5}{ }^{-}-\mathrm{HSO}_{5}{ }^{-}=1,1 \mathrm{~V}, \mathrm{pH} 7\right)$, podendo, em uma série de reações, oxidar novamente $\mathrm{Fe}(\mathrm{II})$ a $\mathrm{Fe}(\mathrm{III})$. Como existem reações competitivas, as quantidades relativas dos reagentes envolvidos constituem um aspecto importante no mecanismo global.

Tabela 12. Atividade catalítica de íons metálicos no processo de oxidação do S(IV) 
Outro fator a ser considerado na velocidade das reações de oxidação de S(IV) catalisadas por metal de transição é a velocidade de substituição do ligante coordenado ao íon metálico e a estabilidade do complexo produzido. Como já mencionado, os íons metálicos de transição podem formar inúmeros complexos com os íons sulfito, bissulfito, sulfato, cloreto, anions orgânicos (Tabela 7), que devem se decompor espontaneamente gerando espécies metálicas reduzidas e o radical $\mathrm{SO}_{3}{ }^{-}$. A influência dos anions no processo pode ocorrer, basicamente, por : 1) o anion atuar como um radical; 2) o anion funcionar como um ligante competitivo para o centro metálico; 3) o anion coordenado acelerar ou inibir o processo de reoxidação da espécie metálica reduzida; 4) efeito de translabilização por coordenação de anions.

Tem sido sugerido na literatura ${ }^{9}$, que alguns anions reagem com os radicais livres produzidos durante o processo, inibindo portanto a reação de oxidação do $\mathrm{S}(\mathrm{IV})$. Por exemplo, o íon $\mathrm{Cl}^{-}$reage rapidamente com o radical $\mathrm{SO}_{4}{ }^{\cdot-}$, podendo inibir o processo global, se reações envolvendo este radical forem determinantes.

No caso de existirem anions que competem com o ligante sulfito, complexos com diferentes estabilidades poderão ser formados (Tabela 7). Deste modo, a estabilidade dos complexos metálicos formados exibe uma certa dependência com os sais a partir dos quais as soluções aquosas foram obtidas.

Ligantes coordenados podem mudar o potencial de oxi-redução das espécies metálicas reduzidas e oxidadas, e portanto afetar a etapa oxidação/redução para o íon metálico. Por exemplo, a velocidade de oxidação do $\mathrm{Fe}(\mathrm{II})$ decresce na presença dos íons: $\mathrm{ClO}_{4}^{-}>\mathrm{NO}_{3}^{-}>$ $\mathrm{Cl}^{-}>\mathrm{Br}^{-}>\mathrm{I}^{-}>\mathrm{SO}_{4}{ }^{2-(95)}$. $\mathrm{O}$ forte efeito inibidor para o íon sulfato tem sido explicado de duas maneiras: a espécie $\mathrm{FeSO}_{4}$ seria menos reativa que o aquocomplexo de $\mathrm{Fe}(\mathrm{II})$, ou poderia haver a formação de espécies diméricas $\mathrm{Fe}^{2+}-\mathrm{SO}_{4}-\mathrm{Fe}^{2+}$. A literatura tem mostrado também o efeito inibitório de anions orgânicos devido à formação de complexos estáveis. A reatividade de complexos de $\mathrm{Cu}$ (II) é substancialmente menor quando ligantes orgânicos estão coordenados, o que foi explicada em termos de mudança do potencial de oxi-redução ${ }^{96}$. Em contraste, observou-se que a cinética de oxidação do $\mathrm{Cr}$ (II) aquoso por $\mathrm{H}_{2} \mathrm{O}_{2}$ é acelerada por um fator de 2 a 5 , na presença de anions orgânicos e inorgânicos ${ }^{97}$. Foi sugerida a formação de espécies com anions coordenados ao $\mathrm{Cr}$ (II), mais facilmente oxidadas que o hexaquocomplexo.

O efeito de translabilização poderá ser induzido por alguns anions, acelerando a substituição de ligantes, e conseqüentemente a decomposição dos complexos será mais rápida. A possível influência dos anions tem sido estudada, utilizando-se diferentes sais para o mesmo metal de transição, ou adicionando-se o anion na forma de sal de alcalino ou alcalino terroso. A atividade catalítica de diferentes sais tem sido relatada na literatura, principalmente, para $\mathrm{Cu}(\mathrm{II}), \mathrm{Mn}(\mathrm{II})$ e $\mathrm{Fe}(\mathrm{III})$. Conklin e Hoffmann ${ }^{98}$ encontraram uma maior velocidade na oxidação do S(IV), quando se utilizou cloreto de cobre em vez de sulfato de cobre. Em contraste, Basset e Parker ${ }^{93}$ reportaram uma atividade catalítica muito mais alta para sulfato de $\mathrm{Mn}$ (II) do que para cloreto de $\mathrm{Mn}$ (II). Por outro lado, Huss e col. ${ }^{20}$ não observaram diferença na velocidade das reações, quando cloreto e sulfato de $\mathrm{Mn}$ (II) foram utilizados. Uma comparação da atividade catalítica de diferentes sais de $\mathrm{Fe}(\mathrm{III})$, indicou que o cloreto de $\mathrm{Fe}$ (III) apresenta a maior atividade, enquanto o sulfato de $\mathrm{Fe}(\mathrm{III})$, a mais baixa?

Um aspecto determinante nas reações de oxidação do S(IV) catalisadas por metais parece ser o $\mathrm{pH}$. As diversas espécies metálicas possíveis de se formarem, bem como as espécies de S(IV), exibem diferentes reatividades. Por exemplo, em um estudo de autoxidação de sulfito catalisada pelo par $\mathrm{Cu}$ (III/II), foi reportado que $\mathrm{HSO}_{3}^{-}$é de 20 a 40 vezes menos reativo que o $\mathrm{SO}_{3}{ }^{2-(99)}$. Ali e col. ${ }^{100}$ reportaram a seguinte ordem de reatividade: $\mathrm{SO}_{3}{ }^{2-}>\mathrm{HSO}_{3}{ }^{-}>$
$\mathrm{SO}_{2} \cdot \mathrm{H}_{2} \mathrm{O}$, em um estudo de oxidação de $\mathrm{S}(\mathrm{IV})$ catalisada por $\mathrm{Co}(\mathrm{III})$. Além disso o $\mathrm{pH}$ afeta a estabilidade dos próprios complexos formados. Alguns autores estudaram a oxidação de S(IV) catalisada por metais em uma faixa de $\mathrm{pH}$ ampla e concluíram que o mecanismo da reação pode inclusive, ser alterado, a depender do $\mathrm{pH}$. Assim, Martin e col. ${ }^{13}$ sugeriram um mecanismo onde não há formação de radicais, para a oxidação de $\mathrm{S}(\mathrm{IV})$ catalisada por $\mathrm{Fe}(\mathrm{III})$, numa faixa de $\mathrm{pH}$ de 0 a 3 , e um mecanismo por radical, para o mesmo processo, numa faixa de $\mathrm{pH}$ de 4 a 7 . Segundo os autores, é impossível formular uma lei de velocidade geral, para um larga faixa de $\mathrm{pH}$, sendo as seguintes equações propostas:

$$
\begin{aligned}
& \text { pH } 0-3-\mathrm{d}[\mathrm{S}(\mathrm{IV})] / \mathrm{dt}=6,0[\mathrm{Fe}(\mathrm{III})][\mathrm{S}(\mathrm{IV})]\left[\mathrm{H}^{+}\right] \\
& \text {pH } 4-\mathrm{d}[\mathrm{S}(\mathrm{IV})] / \mathrm{dt}=1,0 \times 10^{9}[\mathrm{Fe}(\mathrm{III})]^{2}[\mathrm{~S}(\mathrm{IV})] \\
& \text { pH } 5-6-\mathrm{d}[\mathrm{S}(\mathrm{IV})] / \mathrm{dt}=1,0 \times 10^{-3}[\mathrm{~S}(\mathrm{IV})] \\
& \text { pH } 7-\mathrm{d}[\mathrm{S}(\mathrm{IV})] / \mathrm{dt}=1,0 \times 10^{-4}[\mathrm{~S}(\mathrm{IV})]
\end{aligned}
$$

para as condições: $[\mathrm{Fe}(\mathrm{III})]>1,0 \times 10^{-7} \mathrm{~mol} \mathrm{~L}^{-1}$, [S(IV)] $<1,0 \times 10^{-5}$ mol L ${ }^{-1}, \mathrm{~T}=25^{\circ} \mathrm{C}$. Na Tabela 13 , encontram-se os valores de ordens de reação para a oxidação de espécies de $\mathrm{S}(\mathrm{IV})$ catalisadas por $\mathrm{Fe}(\mathrm{II})$, $\mathrm{Fe}(\mathrm{III})$ e Mn(II), obtidas em diversos laboratórios. Mais uma vez se constata que os resultados não são concordantes, também com relação a este aspecto. De uma maneira geral, a velocidade das reações catalisadas por íons metálicos, exibe uma curva dependente do $\mathrm{pH}$, com um máximo em torno de 2 a 4 .

Tabela 13. Dependência da ordem de reação com o pH para oxidação catalítica do S(IV)

\begin{tabular}{cccc}
\hline Espécie & $\mathrm{pH}$ & ordem de reação & Ref. \\
\hline $\mathrm{Fe}^{3+}$ & $0-3$ & 1 & 13 \\
$\mathrm{Fe}^{3+}$ & 4 & 2 & 13 \\
$\mathrm{Fe}^{3+}$ & $5-7$ & 0 ou 2 & 13 \\
$\mathrm{Fe}^{2+}$ & 1,67 & 0,94 & 18 \\
$\mathrm{Fe}^{2+}$ & 2,14 & 1,2 & 18 \\
$\mathrm{Mn}^{2+}$ & $0-3$ & 2 & 14 \\
$\mathrm{Mn}^{2+}$ & $1-4$ & 2 & 18 \\
$\mathrm{Mn}^{2+}$ & $3-6$ & 1 & 12 \\
$\mathrm{Fe}^{3+}$ & 4 & 1 & 12 \\
\hline
\end{tabular}

Com relação aos produtos formados durante as reações de oxidação do S(IV), o acompanhamento destas, pela técnica de cromatografia iônica, mostra que tanto o sulfato, $\left(\mathrm{SO}_{4}^{2-}\right)$, quanto o íon ditionato, $\left(\mathrm{S}_{2} \mathrm{O}_{6}^{2-}\right)$, são possíveis de serem formados. A quantidade relativa dos produtos depende de uma série de fatores tais como: presença de íons metálicos, concentrações relativas da espécie inicial de S(IV) e do íon metálico, presença de anions, pH da solução. Alguns autores observaram um aumento da concentração de ditionato, para a reação conduzida na presença de íons metálicos, em comparação com os resultados obtidos sem a presença destes ${ }^{101}$. Foi observado também que a concentração do ditionato depende do íon metálico usado como catalisador, sendo reportado as seguintes quantidades de ditionato para diferentes íons metálicos: Mn(IV) 49\%, Fe(III) 39\%, Co(III) $15 \%, \mathrm{Ni}$ (III) $9 \%{ }^{102}$.

O efeito do oxigênio na formação do ditionato não está esclarecido. Na reação catalisada por $\mathrm{Fe}(\mathrm{III}), \mathrm{Kraft}^{103}$ encontrou uma maior quantidade de ditionato, para a reação conduzida na presença de oxigênio do que na ausência. Já Faust e col. ${ }^{104}$ e Grgic e col. ${ }^{12}$ não encontraram a presença de ditionato para a reação catalisada por $\mathrm{Fe}(\mathrm{III}) \mathrm{em}$ presença de ar. Brandt e van Eldik ${ }^{105}$ observaram que a concentração de ditionato é dependente da relação [Fe(III)]:[S(IV)], do pH da solução, mas independente da concentração inicial de oxigênio. 
De acordo com Veprek-Siska e col. ${ }^{106}$, a reatividade do complexo metal-sulfito formado na etapa inicial do processo, é o principal fator que influencia os produtos da reação. Complexos com ligação metal-sulfito através do átomo de enxofre resultam na formação somente de sulfato. Já quando o íon sulfito se liga ao metal via dois átomos de oxigênio, a ligação $\mathrm{S}-\mathrm{S}$ é favorecida e os produtos de oxidação serão sulfato e ditionato.

\section{Reação de oxidação do S(IV) na presença de ozônio}

Embora o ozônio reaja com $\mathrm{SO}_{2}$ muito lentamente na fase gasosa, a reação na fase aquosa é bastante rápida. A concentração de ozônio na fase aquosa, em equilíbrio com uma concentração de $40 \mathrm{ppb}$ na fase gasosa, é $5 \times 10^{-10} \mathrm{~mol} \mathrm{~L}^{-1}$ a $25^{\circ} \mathrm{C}\left(\boldsymbol{H}=1,3 \times 10^{-2} \mathrm{~mol} \mathrm{~L}^{-1} \mathrm{~atm}^{-1}\right)$. Tal concentração é cerca de 6 ordens de grandeza menor que a do oxigênio dissolvido, porém a oxidação do $\mathrm{S}(\mathrm{IV})$ por $\mathrm{O}_{3}$ pode se tornar importante sob determinadas condições, devido a constantes de velocidade mais altas. Na Figura 6 estão representadas as velocidades estimadas para a oxidação do $\mathrm{S}(\mathrm{IV})$ por $\mathrm{H}_{2} \mathrm{O}_{2}$ e $\mathrm{O}_{3}$, em função do $\mathrm{pH}$. No caso do ozônio, a dependência com o pH mostra um decréscimo na velocidade de produção de $\mathrm{S}(\mathrm{VI})$, quando o $\mathrm{pH}$ diminui, em contraste com $\mathrm{H}_{2} \mathrm{O}_{2}$, cuja taxa de oxidação do S(IV) varia muito pouco em função do $\mathrm{pH}$.

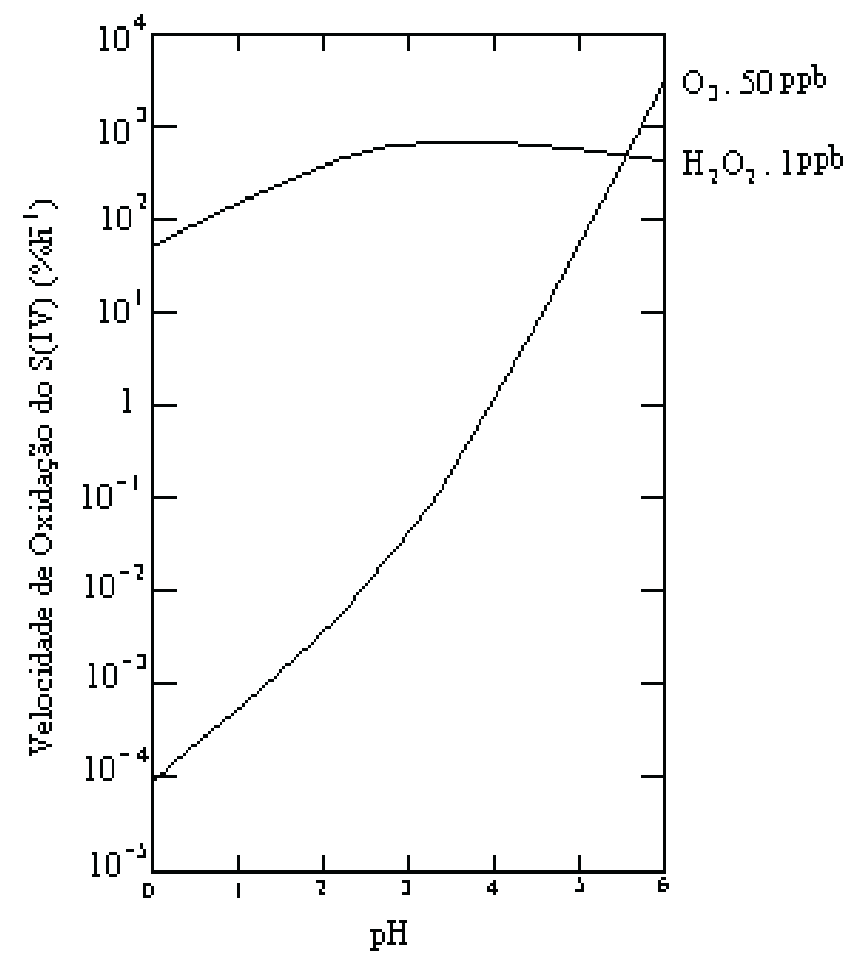

Figura 6. Velocidades estimadas para a reação de oxidação do $S(I V)$ por $\mathrm{H}_{2} \mathrm{O}_{2}$ e $\mathrm{O}_{3}$ (adaptado da Ref. 14)

O mecanismo da oxidação de S(IV) na presença de ozônio permanece incerto: uma simples reação de transferência de oxigênio tem sido discutida, bem como um ataque eletrofílico do ozônio ao átomo de enxofre. Trabalhos mais recentes reportados na literatura propõem também um mecanismo por radical livre. A reação é descrita na literatura como sendo de primeira ordem em relação a concentração de S(IV) e de ozônio. Botha e colaboradores ${ }^{11}$ sugerem o seguinte esquema de equações para a oxidação de $\mathrm{S}(\mathrm{IV})$ por ozônio em solução aquosa:

Decomposição do $\mathrm{O}_{3}$ :

$2 \mathrm{O}_{3}+\mathrm{OH}^{-} \rightarrow \mathrm{O}_{2}^{\cdot}+\mathrm{HO}^{\bullet}+2 \mathrm{O}_{2}$
Formação de $\mathrm{HSO}_{5} \cdot$

$\mathrm{HSO}_{3}{ }^{-}+\mathrm{HO}^{\cdot} \rightarrow \mathrm{HSO}_{3}{ }^{-}+\mathrm{OH}^{-}$

$\mathrm{HSO}_{3}^{\cdot}+\mathrm{O}_{2} \rightarrow \mathrm{HSO}_{5}^{\cdot}$

Oxidação do S(IV):

$\mathrm{HSO}_{5}^{-}+\mathrm{SO}_{2} \cdot \mathrm{H}_{2} \mathrm{O} \rightarrow \mathrm{HSO}_{5}^{-}+\mathrm{HSO}_{3}^{-}+\mathrm{H}^{+}$

$\mathrm{HSO}_{5}^{-}+\mathrm{SO}_{2} \cdot \mathrm{H}_{2} \mathrm{O} \rightarrow 3 \mathrm{H}^{+}+2 \mathrm{SO}_{4}^{2-}$

$\mathrm{HSO}_{5}{ }^{-}+\mathrm{HSO}_{3}^{-} \rightarrow \mathrm{HSO}_{5}^{-}+\mathrm{HSO}_{3} \cdot$

$\mathrm{HSO}_{5}^{-}+\mathrm{HSO}_{3}^{-} \rightarrow 2 \mathrm{H}^{+}+2 \mathrm{SO}_{4}^{2-}$

$\mathrm{HSO}_{5}{ }^{-}+\mathrm{SO}_{3}^{2-} \rightarrow \mathrm{SO}_{5}^{2-}+\mathrm{HSO}_{3} \cdot$

$\mathrm{HSO}_{5}^{-}+\mathrm{SO}_{3}^{2-} \rightarrow \mathrm{H}^{+}+2 \mathrm{SO}_{4}^{2-}$

A influência dos íons metálicos no processo de oxidação de S(IV), em presença de ozônio, tem sido pouco investigada e os resultados descritos na literatura não são concordantes. Em alguns trabalhos desenvolvidos em diferentes laboratórios ${ }^{11,14}$, não se observou influência dos íons metálicos $\left(\mathrm{Cr}^{3+}, \mathrm{Fe}^{2+}, \mathrm{Fe}^{3+}, \mathrm{Mn}^{2+}\right.$ e $\left.\mathrm{Cu}^{2+}\right)$, enquanto Harrison e col. ${ }^{27}$ encontraram efeito catalítico, com a adição dos íons $\mathrm{Mn}^{2+}$ e $\mathrm{Fe}^{3+}$. Este último foi realizado numa faixa de $\mathrm{pH}$ de 4 a 6 , sendo que a atividade catalítica foi máxima em $\mathrm{pH}$ próximo a 4,5. Foi sugerido, que a catálise por $\mathrm{Fe}^{3+}$ e $\mathrm{Mn}^{2+}$ poderia ocorrer através da formação inicial de um complexo metal-sulfito, seguida de uma reação com ozônio, para formar um complexo ternário ${ }^{14}$ :

$\mathrm{Fe}^{3+}+\mathrm{H}_{2} \mathrm{O}=\mathrm{FeOH}^{2+}+\mathrm{H}^{+}$

$\mathrm{FeOH}^{2+}+\mathrm{HSO}_{3}^{-}=\mathrm{Fe}(\mathrm{OH}) \mathrm{SO}_{3}{ }^{\circ}+\mathrm{H}^{+}$

$\mathrm{Fe}(\mathrm{OH}) \mathrm{SO}_{3}{ }^{\circ}+\mathrm{O}_{3}=\mathrm{Fe}(\mathrm{OH}) \mathrm{SO}_{3} \mathrm{O}_{3}^{\circ}$

$\mathrm{Fe}(\mathrm{OH}) \mathrm{SO}_{3} \mathrm{O}_{3}{ }^{\circ} \rightarrow \mathrm{FeOH}^{2+}+\mathrm{SO}_{4}{ }^{2-}+\mathrm{O}_{2}$

\section{Reação de oxidação de $\mathrm{S}(\mathrm{IV})$ na presença de $\mathrm{NO}_{2}$}

Sistemas aquosos contendo S(IV) podem ser oxidados por espécies de N(III) e N(IV), na forma de nitrito, ácido nitroso e dióxido de nitrogênio aquoso. Em solução ácida, o ácido nitroso oxida S(IV), de acordo com as seguintes reações ${ }^{14}$ :

$$
\begin{aligned}
& 2 \mathrm{HNO}_{2}+\mathrm{H}_{2} \mathrm{O} . \mathrm{SO}_{2} \rightarrow \mathrm{SO}_{4}^{2-}+2 \mathrm{NO}+2 \mathrm{H}^{+}+\mathrm{H}_{2} \mathrm{O} \\
& \Delta \mathrm{G}^{\mathrm{o}}=-160 \mathrm{~kJ} \mathrm{~mol}^{-1} \\
& 2 \mathrm{HNO}_{2}+2 \mathrm{H}_{2} \mathrm{O} . \mathrm{SO}_{2} \rightarrow 2 \mathrm{SO}_{4}^{2-}+\mathrm{N}_{2} \mathrm{O}+4 \mathrm{H}^{+}+\mathrm{H}_{2} \mathrm{O} \\
& \Delta \mathrm{G}^{\mathrm{o}}=-431 \mathrm{~kJ} \mathrm{~mol}^{-1}
\end{aligned}
$$

Na Figura 7, estão representadas as velocidades estimadas para a oxidação do $\mathrm{S}(\mathrm{IV})$ por $\mathrm{NO}_{2}$ e $\mathrm{HNO}_{2}$, em função do $\mathrm{pH}$. Assim como no caso do ozônio, na presença destes oxidantes, a velocidade de oxidação se torna mais elevada para valores mais altos de $\mathrm{pH}$.

Oblath e col. ${ }^{107}$ estudaram a reação entre o íon bissulfito e o íon nitrito ( $\mathrm{pH} 4,5$ - 7), e propuseram um mecanismo com formação de espécies intermediárias contendo ligação S-N. O principal composto formado durante o processo foi a hidroxilaminadisulfonato (HADS), sendo o seguinte esquema de reações sugerido:

$\mathrm{NO}_{2}^{-}+\mathrm{H}^{+}=\mathrm{HONO}$

$\mathrm{HONO}+\mathrm{HSO}_{3}^{-} \rightarrow \mathrm{NOSO}_{3}^{-}+\mathrm{H}_{2} \mathrm{O}$

$\mathrm{ONSO}_{3}^{-}+\mathrm{HSO}_{3}^{-} \rightarrow \mathrm{HON}\left(\mathrm{SO}_{3}\right)_{2}^{2-}$

$2 \mathrm{HSO}_{3}^{-}=\mathrm{S}_{2} \mathrm{O}_{5}^{2-}+\mathrm{H}_{2} \mathrm{O}$

$\mathrm{HONO}+\mathrm{S}_{2} \mathrm{O}_{5}^{2-} \rightarrow \mathrm{HON}\left(\mathrm{SO}_{3}\right)_{2}^{2-}$

$\mathrm{HON}\left(\mathrm{SO}_{3}\right)_{2}{ }^{2-}+\mathrm{HSO}_{3}^{-} \rightarrow \mathrm{N}\left(\mathrm{SO}_{3}\right)_{3}{ }^{3-}+\mathrm{H}_{2} \mathrm{O}$

$\mathrm{HON}\left(\mathrm{SO}_{3}\right)_{2}^{2-}+\mathrm{SO}_{3}{ }^{2-} \rightarrow \mathrm{HN}\left(\mathrm{SO}_{3}\right)_{2}^{2-}+\mathrm{SO}_{4}{ }^{2-}$

$\mathrm{HON}\left(\mathrm{SO}_{3}\right)_{2}^{2-}+\mathrm{H}^{+}+\mathrm{H}_{2} \mathrm{O} \rightarrow \mathrm{HONHSO}_{3}^{-}+\mathrm{HSO}_{4}^{-}$ 


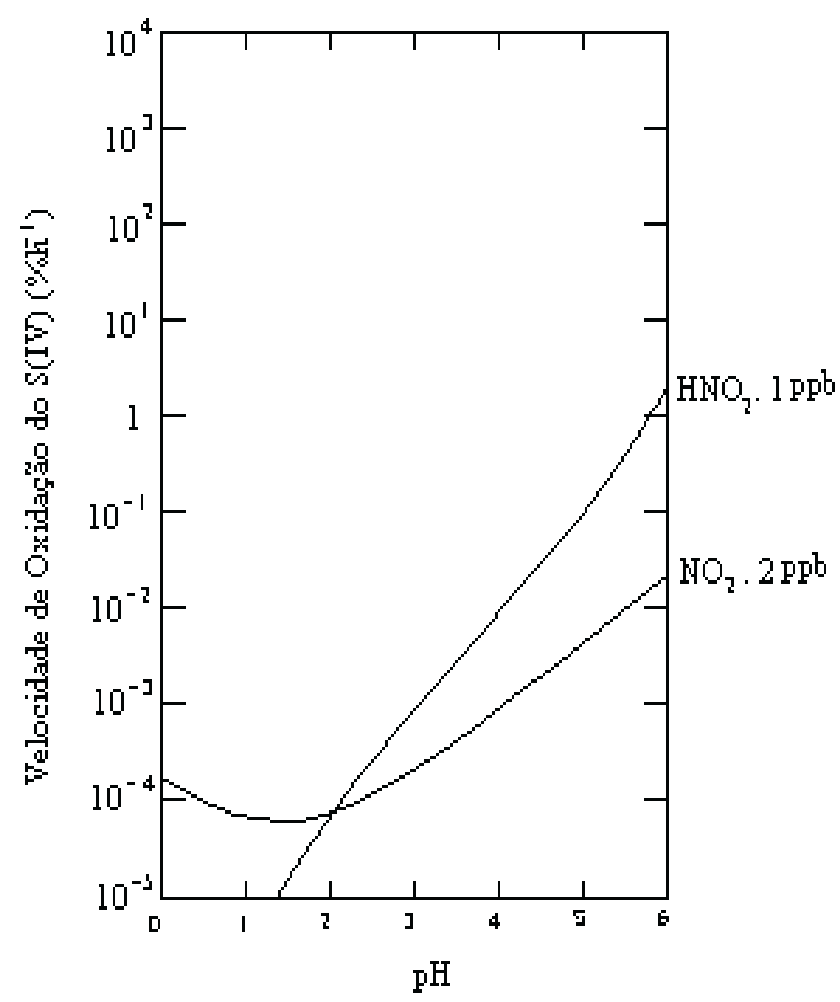

Figura 7. Velocidades estimadas para a reação de oxidação do $S(I V)$ por $\mathrm{NO}_{2}$ e $\mathrm{HNO}_{2}$ (adaptado da Ref.14)

Esta seqüência de reações tem sido considerada por vários autores em seus trabalhos ${ }^{108}$.

A reação entre dióxido de nitrogênio e solução aquosa de sulfito foi também estudada através da espectroscopia Raman, na presença e na ausência de oxigênio ${ }^{109}$. Os produtos observados incluíram os íons nitrito, sulfato e ditionato. $\mathrm{O}$ mecanismo sugerido indica a formação inicial do radical $\mathrm{SO}_{3} \cdot$ pela reação do dióxido de nitrogênio com o íon sulfito (eq. 70):

$\mathrm{NO}_{2}+\mathrm{SO}_{3}^{2-} \rightarrow \mathrm{NO}_{2}^{-}+\mathrm{SO}_{3}^{-}$

Na presença de oxigênio, novos radicais em cadeia são formados, e a oxidação se completa:

$\mathrm{SO}_{3}{ }^{-}+\mathrm{O}_{2} \rightarrow \mathrm{SO}_{5}^{\cdot}$

$\mathrm{SO}_{5}^{--}+\mathrm{SO}_{3}^{2-} \rightarrow \mathrm{SO}_{5}^{2-}+\mathrm{SO}_{3}^{*}$

$\mathrm{SO}_{5}^{*-}+\mathrm{SO}_{3}^{2-} \rightarrow \mathrm{SO}_{4}^{2-}+\mathrm{SO}_{4}^{\cdot}$

$\mathrm{SO}_{4}^{--}+\mathrm{SO}_{3}^{2-} \rightarrow \mathrm{SO}_{4}^{2-}+\mathrm{SO}_{3}^{-}$

$\mathrm{SO}_{5}^{2-}+\mathrm{H}^{+}=\mathrm{HSO}_{5}$

$\mathrm{HSO}_{5}^{-}+\mathrm{SO}_{3}^{2-} \rightarrow \mathrm{SO}_{4}^{2-}+\mathrm{SO}_{4}^{2-}$

$\mathrm{Na}$ ausência de oxigênio, foi observada a formação do íon ditionato, atribuída à reação:

$\mathrm{SO}_{3}{ }^{\cdot-}+\mathrm{SO}_{3}^{\cdot-} \rightarrow \mathrm{S}_{2} \mathrm{O}_{6}{ }^{2-}$

Relativamente poucos estudos sobre o efeito dos íons metálicos nas reações de oxidação de S(IV), em presença de óxidos de nitrogênio, têm sido reportados. Martin e col. ${ }^{110}$ observaram que a adição de $\mathrm{Fe}^{3+}$ ou $\mathrm{Mn}^{2+}$ ao sistema, em presença do íon nitrito, não alterou a velocidade de oxidação do S(IV). Da mesma forma, Ellison e col. ${ }^{111}$

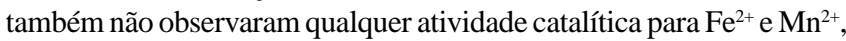
na oxidação de $\mathrm{S}(\mathrm{IV})$ na presença de $\mathrm{NO}_{2}$. Já, nos estudos realizados por $\mathrm{NaSh}^{28}$, relativos à influência do $\mathrm{Fe}^{2+}, \mathrm{Mn}^{2+} \mathrm{e} \mathrm{Cu}^{2+}$, na reação de oxidação do $\mathrm{S}(\mathrm{IV})$ por dióxido de nitrogênio, os resultados indicaram forte efeito catalítico para $\mathrm{Fe}^{2+}$, não se observando, porém, efeito catalítico para $\mathrm{Cu}^{2+}$ e $\mathrm{Mn}^{2+}$.

\section{IMPACTO AMBIENTAL}

As principais conseqüências para o ambiente, decorrentes dos diversos processos de oxidação das espécies de S(IV) na troposfera, correspondem ao aumento de acidez da fase aquosa, e à formação de sulfato particulado no aerossol atmosférico.

\section{Chuva Ácida}

Substâncias emitidas para a atmosfera podem retornar quimicamente transformadas à superfície, via processos de deposição seca (sem a participação da fase líquida), ou deposição úmida. A deposição úmida ocorre através de chuva, orvalho, neblina e neve. O termo chuva ácida tem sido usado freqüentemente como uma expressão para todos os processos de deposição úmida ${ }^{112,113}$.

$\mathrm{O}$ interesse científico na deposição úmida tem aumentado como conseqüência de prejuízos ecológicos e econômicos, tais como danos às florestas, à flora e fauna aquática, e aos materiais de construção. $\mathrm{O}$ valor do $\mathrm{pH}$ da água de chuva se encontra mais comumente na faixa 4,5 - 5,6, considerando-se o conteúdo natural de $\mathrm{CO}_{2}$ da atmosfera e a contribuição de compostos de enxofre ${ }^{114}$.

As mais importantes substâncias ácidas inorgânicas na atmosfera são $\mathrm{H}_{2} \mathrm{SO}_{4}$ e $\mathrm{HNO}_{3}$ (Tabela 14). Dos ácidos orgânicos, os presentes em maior quantidade são o ácido fórmico e o ácido acético ${ }^{119,120}$. A contribuição relativa de compostos ácidos na acidez da precipitação depende do tipo da fase aquosa e local de coleta (Tabela 15). A contribuição de ácidos orgânicos pode dominar em regiões tropicais e equatoriais. Fontes naturais destes ácidos ou de seus precursores são as plantas, porém atividades antropogênicas incluindo emissão por tráfego, também contribuem para a formação destes. A contribuição máxima de $\mathrm{H}_{2} \mathrm{SO}_{4}$ e $\mathrm{HNO}_{3}$ em precipitação ácida nos EUA foi de $73 \%$ e $31 \%$ (verão), e de $59 \%$ e $61 \%$ (inverno), respectivamente. Contribuições mais altas de $\mathrm{HNO}_{3}$ durante o inverno são devidas a emissões mais altas de $\mathrm{NO}_{\mathrm{x}}$, provenientes de processos de

Tabela 14. Percentagem de contribuição ácida na água de chuva (HX $=\mathrm{HCl}, \mathrm{H}_{3} \mathrm{PO}_{4}$, ou ácidos orgânicos)

\begin{tabular}{ccclc}
\hline$\% \mathrm{H}_{2} \mathrm{SO}_{4}$ & $\% \mathrm{HNO}_{3}$ & $\% \mathrm{HX}$ & Local & Ref. \\
\hline 65 & 17 & 18 & Alaska & 115 \\
38,9 & 33,6 & 27,4 & Los Angeles, CA & 114 \\
73 & 31 & & EUA (NE)-verão & 116 \\
59 & 61 & & EUA (NE)-inverno & 116 \\
19 & 12 & 63 & Brasil & 117 \\
70 & 30 & & Inglaterra & 118 \\
\hline
\end{tabular}

Tabela 15. Concentrações de $\mathrm{H}_{2} \mathrm{SO}_{4}$ e $\mathrm{HNO}_{3}$ na fase aquosa atmosférica

\begin{tabular}{lcccc}
\hline Local & $\begin{array}{c}\mathrm{H}_{2} \mathrm{SO}_{4} \\
(\mu \mathrm{mol} \mathrm{L}-1)\end{array}$ & $\begin{array}{c}\mathrm{HNO}_{3} \\
\left(\mu \mathrm{mol} \mathrm{L}^{-1}\right)\end{array}$ & $\mathrm{pH}$ & Ref. \\
\hline Amazônia (chuva) & 4,5 & 5,5 & 4,6 & 117 \\
$\begin{array}{l}\text { Pasadena, Califórnia } \\
\text { (neblina) }\end{array}$ & 2530 & 1200 & 2,25 & 121 \\
Ithaca, NY (chuva) & 57,1 & 44,4 & 3,84 & 122 \\
Patros, Grécia (chuva) & 38 & 57 & $4,12-8,76$ & 123 \\
Pune, Índia (nuvem) & 15,6 & 29 & 6,9 & 124 \\
\hline
\end{tabular}


combustão para o aquecimento. Já na Europa, durante o inverno, a concentração de $\mathrm{H}_{2} \mathrm{SO}_{4}$ é duas vezes maior que a de $\mathrm{HNO}_{3}$.

$\mathrm{O}$ estudo da deposição úmida no Recôncavo Baiano, realizado a partir de amostras de chuva coletadas em quatro estações, indica uma faixa de valores de $\mathrm{pH}$ de 4,1 a 7,1, com valor mediano de $5,0^{125}$. Na Tabela 16 podem ser vistos os resultados referentes à coleta nas quatro estações. Este estudo concluiu que 16,5\% das amostras de chuva coletadas em Salvador e $23 \%$ das coletadas em Pedra do Cavalo, apresentaram valores de $\mathrm{pH}$ abaixo de 5,0, porém nas estações da CIBEB ( $2 \mathrm{Km}$ do Polo Petroquímico) e da CEPLAC, $60 \%$ tem $\mathrm{pH}$ abaixo de 5,0, indicando que as mesmas recebem chuva ligeiramente ácida.

Tabela 16. Valores de $\mathrm{pH}$ para água de chuva (média ponderada), no Recôncavo Baiano,(1986-1987) 125

\begin{tabular}{lc}
\hline Estação & $\mathrm{pH}$ (média ponderada) \\
\hline Ondina, Salvador (BA) & 5,3 \\
Pedra do Cavalo, Cachoeira (BA) & 5,1 \\
CEPLAC, São Sebastião do Passé (BA) & 4,8 \\
CIBEB, Camaçarí (BA) & 4,9 \\
\hline
\end{tabular}

Além de poluir rios e lagos, destruindo a flora e a fauna aquática, a chuva ácida se infiltra no solo, liberando metais potencialmente tóxicos tais como $\mathrm{Al}, \mathrm{Pb}, \mathrm{Cd}$, que podem se introduzir na cadeia alimentar. A extensão na qual a precipitação ácida afeta uma determinada área, depende significativamente da composição do solo: áreas fortemente afetadas são as que contém granito ou quartzo, já que estes tem pequena capacidade de neutralização; em contraste, solos contendo carbonato de cálcio, podem neutralizar a acidez de modo eficiente. Lagos com características ácidas apresentam concentrações elevadas de $\mathrm{Al}^{3+}$; esta combinação, acidez e altas concentrações do metal, é responsável pela devastação de peixes e plantas aquáticas.

Nos últimos anos, tem se verificado uma relação direta entre danos à vegetação e precipitação ácida. O fenômeno de destruição de florestas foi observado pela primeira vez, em larga escala, na Alemanha. Florestas com elevadas altitudes são as mais afetadas pela precipitação ácida, provavelmente por estarem mais expostas à base de nuvens baixas, onde a acidez é mais concentrada. Os prejuízos da chuva ácida incluem também o ataque a diversos materiais de construção, tais como aço, tintas, plásticos, cimentos e vários tipos de pedra. Assim é que construções históricas, como: o Taj Mahal, na Índia, as obras da Civilização Maia, no México, o Coliseu, em Roma e outros tem sofrido os efeitos deste fenômeno. Estudo realizado na Grécia, indicou que o Paternon Atenas se deteriorou nos últimos 25 anos mais que nos 2400 anos anteriores, devido aos efeitos da chuva ácida.

Os ácidos presentes na chuva ácida podem ser formados durante o transporte de massas de ar que contém os poluentes primários. Sendo assim, a chuva ácida pode ser gerada em locais bem distantes das fontes poluidoras. Milhares de lagos mortos na Escandinávia se encontram neste estado pela ação de chuva ácida, gerada por poluentes originários da Alemanha, França e Grã-Bretanha. De maneira similar, a precipitação ácida no Canadá é consequência da emissão do dióxido de enxofre em Ohio Valley, nos EUA. Por outro lado, a reação de $\mathrm{SO}_{2}$ com formaldeído, formando o ácido hidroximetanosulfônico $^{126,127,128}$, constitui uma forma de estabilização de S(IV), resultando num maior tempo de residência no ambiente.

\section{Sulfato particulado em aerossóis atmosféricos}

Outro fenômeno associado à transformação do $\mathrm{SO}_{2}$ na atmosfera é a formação do aerossol de sulfato, na forma de gotas de $\mathrm{H}_{2} \mathrm{SO}_{4}$ ou partículas de sais. Além do $\mathrm{H}_{2} \mathrm{SO}_{4}$, que se encontra altamente hidratado, os principais sulfatos presentes no aerossol atmosférico são: $\mathrm{NH}_{4} \mathrm{HSO}_{4},\left(\mathrm{NH}_{4}\right)_{2} \mathrm{SO}_{4},\left(\mathrm{NH}_{4}\right)_{3} \mathrm{H}\left(\mathrm{SO}_{4}\right)_{2}, \mathrm{MgSO}_{4}$ e $\mathrm{CaSO}_{4}$.

As partículas do aerossol de sulfato exibem diâmetro $<10 \mu \mathrm{m}$ $\left(\mathrm{PM}_{10}\right)$, sendo perigosas para a saúde, pois penetram profundamente nos pulmões, causando e agravando problemas respiratórios. Além disso, o aerossol de sulfato provoca degradação de visibilidade, como resultado do fenômeno da dispersão da luz. A química atmosférica do $\mathrm{SO}_{2}$ tem um papel importante, não só na formação de $\mathrm{PM}_{10}$, como também na formação de partículas finas. Essencialmente, a maior parte do sulfato particulado tem diâmetro menor que $2,5 \mathrm{~mm}$, contribuindo com mais da metade do material particulado fino, presente em áreas urbanas.

Partículas com diâmetro aerodinâmico (D) $\leq 2,5 \mathrm{~mm}$ contribuem predominantemente para o espalhamento do tipo Mie $(\mathrm{D} \cong \lambda$ da luz $)^{1}$, sendo a mais importante causa da diminuição de visibilidade. A umidade relativa é um fator que influencia o grau de degradação de visibilidade associado ao sulfato particulado. Hering e col ${ }^{129}$ encontraram duas faixas discretas de tamanho para o sulfato particulado presente na atmosfera de Los Angeles: 0,54 $\pm 0,07 \mu \mathrm{m}$ e $0,20 \pm 0,02 \mu \mathrm{m}$. A primeira distribuição de tamanho é decorrente do processo de conversão em fase líquida, e estas partículas estão associadas com o máximo espalhamento da luz. A distribuição de tamanho menor é resultante do processo de conversão homogênea, em fase gasosa. Estas partículas estão também associadas com o espalhamento da luz, mas não na mesma extensão das partículas formadas pelo processo heterogêneo, em fase líquida. Com o aumento da umidade relativa, a reação em fase líquida se torna mais importante, aumentando a degradação de visibilidade. Portanto, a química de conversão do $\mathrm{SO}_{2}$ gasoso a sulfato particulado, ocorrendo em nuvens, nevoeiros, ou outras condições de alta umidade, contribui significativamente para a redução de visibilidade.

\section{CONSIDERAÇÕES FINAIS}

A água atmosférica (chuva, nuvem, neblina) pode ser vista como uma câmara, na qual várias reações envolvendo a oxidação do S(IV) ocorrem simultaneamente, e cada reação, em particular, pode exercer influência sobre as outras. A contribuição individual de cada rota no processo global da oxidação do S(IV) depende de diversas condições presentes na atmosfera.

O efeito da degradação ambiental decorrente da oxidação do $\mathrm{S}(\mathrm{IV})$ tem aumentado o interesse sobre o assunto, gerando um número razoável de trabalhos. Vários estudos têm sido realizados em laboratório, visando avaliar a influência de íons metálicos e espécies oxidantes, na oxidação do S(IV). Entretanto, pouco foi relatado até o momento, com relação à influência de íons, presentes na atmosfera, tais como $\mathrm{Cr}(\mathrm{VI}), \mathrm{Cr}(\mathrm{III})$ e $\mathrm{Cd}(\mathrm{II})$. Mesmo para os sistemas mais estudados, como por exemplo a oxidação catalítica por Fe(III) e $\mathrm{Mn}(\mathrm{II})$, existem divergências na literatura, com relação a diversos aspectos da reação, especialmente quanto aos mecanismos de reação e valores de constantes de velocidade. A oxidação de espécies de S(IV) catalisada por íons metálicos, parece seguir mecanismos de reação complexos, os quais são provavelmente muito sensíveis às condições individuais de cada experimento. Com relação ao estudo da oxidação catalítica do S(IV) em presença de ozônio ou dióxido de nitrogênio, verifica-se que o número de trabalhos é relativamente baixo, existindo dúvidas com relação à importância dessas espécies, quando em concentrações atmosféricas.

Em muitos dos trabalhos citados nessa revisão, observa-se que as concentrações das espécies metálicas e oxidantes estão bem acima das faixas encontradas normalmente na fase aquosa atmosférica. Os resultados, portanto, poderão não retratar processos reais que 
ocorrem na atmosfera. Sendo assim, seria importante que outros trabalhos fossem desenvolvidos, procurando se aproximar o máximo de condições existentes na atmosfera, a fim de que os resultados possam contribuir de maneira mais efetiva para o entendimento da química atmosférica do enxofre.

\section{AGRADECIMENTOS}

Ao professor Dr. José Oscar Nogueira Reis e à professora Dra. Janete Jane Fernandes Alves pelas valiosas sugestões, à Dra. Luciana Almeida Silva e a Clodovil Alves Cabral Neto pela ajuda na elaboração das figuras deste texto e ao CADCT/SEPLANTEC-BA.

\section{REFERÊNCIAS}

1. Eatough, D.J.; Caka, F.M.; Farber, R.J.; Isr. J. Chem. 1994, 34, 301.

2. Finlayson-Pitts, B.J.; Pitts, J.N.Jr.; Atmospheric Chemistry: Fundamentals and Experimental Techniques, John Wiley \& Sons: New York, 1986.

3. Coffer III, W. R.; Schryer, D. R.; Rogowski, R. S.; Atmos. Environ. 1981, 15,1281 .

4. Junkermann, W.; Roedel, W.; Atmos. Environ. 1985, 19, 1206.

5. Junkermann, W.; Roedel, W.; Atmos. Environ. 1983, 17, 2549.

6. Pandis, S. N.; Seinfeld, J. H.; Atmos. Environ.1992, 26A, 2509.

7. Luria, M.; Sievering, H.; Atmos. Environ. 1991, 25A, 1489.

8. Saxena, P.; Seigneuer, C.; Atmos. Environ. 1987, $21,807$.

9. Brandt, C.; van Eldik, R.; Chem. Rev.1995, 95, 119.

10. Clarke, A. G.; Radojevic, M.; Atmos. Environ. 1983, 17, 617.

11. Botha, C. F.; Hahn, J.; Pienaar, J. J.; van Eldik, R.; Atmos. Environ. 1994, $28,3207$.

12. Grgic, I.; Hudnik, V.; Bizjak, M.; Levec, J.; Atmos. Environ. 1991, 25A, 1591.

13. Martin, L. R.; Hill, M. W.; Tai, A. F.; Good, T. W.; J. Geophys. Res. 1991, 96b(D2), 3085

14. Martin, L. R. Em Acid Precipitation Series; Calvert, J. G., ed.; Butterworth Publishers: Boston, 1984, cap. 2, pp. 63-100; Hoffmann, M. R. \& Jacob, D. J. Em Acid Precipitation Series; Calvert, J. G., ed.; Butterworth Publishers: Boston, 1984, cap. 3, pp. 101-172.

15. Silva, L. A.; Correa, S. J.; Martins, C. R.; de Andrade, J. B.; Quim. Nova 1998, 21, 151.

16. Cox, X. B.; Linton, R. W.; Miguel, A. H.; de Andrade, J. B.; Atmos. Environ. 1986, 20, 1139.

17. Bengtsson, S.; Bjerle, I.; Chem. Eng. Sci. 1975, 30, 1429.

18. Tsunogai, S.; Geochem. J. 1971, 5, 175.

19. Barrie, L.; Georgii, H. W.; Atmos. Environ. 1976, 10, 743.

20. Huss, A., Jr.; Lim, P. K.; Eckert, C.A.; J. Phys. Chem. 1982, 86, 4224.

21. Boyce, S. D.; Hoffmann, M. R.; Hong, P. A.; Moberly, L. M.; Environ. Sci. Technol. 1983, 17, 602.

22. Ulrich, R. K.; Rochelle, G.T.; Prada, R. E.; Chem. Eng. Sci. 1986, 41, 2183.

23. Reda, M.; Water Science Technology 1988, 20, 45.

24. Brandt, C.; Fábián, I.; van Eldik, R.; Inorg. Chem. 1994, 33, 687.

25. Brandt, C.; Elding, L. I.; Atmos. Environ. 1998, 32, 797.

26. Pezza, H. R.; Lopes, C. F. F.; Suárez-Iha, M. E. V.; Coichev, N.; Quim Nova 1999, 22, 529.

27. Harrison, H.; Larson, T. V.; Monkman, C. S.; Atmos. Environ. 1982, 16, 1039.

28. Nash, T.; Atmos. Environ.1979, 13, 1149.

29. Ellison, T. K.; Eckert, C. A.; J. Phys. Chem. 1984, 88, 2335.

30. Cardoso, A. A.; Pitombo, L. R. M.; Quim. Nova 1992, 15, 219.

31. Möller, D.; Atmos. Environ. 1984, 18, 29.

32. Cullis, C. F.; Hischeler, M. M.; Atmos. Environ. 1980, 14, 1263

33. Williams, M. L.; Broughton, G. F. J.; Bower, J. S.; Drury, V. J.; Lilley, K.; Powell, K.; Rogers, F. S. M.; Stevenson, K. J.; Atmos. Environ. 1988, 22, 2819.

34. EPA. National air quality and emissions trends report 1984; Publication No. EPA-450/4-86-001, U.S. Environmental Protection Agency: Research Triangle Park, 1986.

35. Tanner, R. L.; Miguel, A. H.; de Andrade, J. B.; Gaffney, J. S.; Streit, G. E.; Environ. Sci. Technol. 1988, 22, 1026.

36. Alonso, C. D.; Godinho, R.; Quim. Nova, 1992, 15, 126.

37. Lefhon, A.S.; Husar, J. D.; Husar, R.B.; Atmos. Environ. 1999, 33, 3435

38. Logan, J. A.; J. Geophys. Res. 1983, 88, 10785.

39. Neftel, A.; Beer, J.; Oeschger, H.; Zürcher, F.; Finkel, R. C.; Nature 1985, 314,611 .
40. Environmental Data Report 1993-1994, United Nations Environmental Programme; Prepared for UNEP by the GEMS Monitoring and Assessment Research Centre, London, UK, in cooperation with the World Resources Institute, Washington, DC, UK Departament of Environment, London; Blackwell Publishers: Oxford, UK, 1993; pp. 44-46.

41. Global Institute of Ecology and Climate, USSR, Academy of Science, Moscow, Russia. Cited in ref. 40.

42. Dignon, J.; Hameed, S.; J. Air Pollut. Control. Assoc. 1989, 39,180.

43. Graedel, T. E.; Chemical Compounds in the Atmosphere, Academic Press: New York, 1978, p 16.

44. Andrade, M. V. A.; Nunes, F. M. N.; Carvalho, A. S.; de Andrade, J. B.; Resumos da $22^{a}$ Reunião Anual da Sociedade Brasileira de Química, Poços de Caldas, Brasil, 1999.

45. Nriagu, J. O.; Nature 1979, 279, 409.

46. Loranger, S.; Zayed, J.; Atmos. Environ. 1994, 27, 1347.

47. Nriagu, J. O.; Nature 1989, 338, 47.

48. Lantzy, R. J.; Mackenzie, F. T.; Geochim. Cosmochim. Acta 1979, 43, 511.

49. Bertine, K. K.; Golberg, E. D.; Science 1971, 173, 233.

50. de Andrade, J. B.; Macedo, M. A.; Korn, M.; Oliveira,E.; Gennari, R. F.; Toxicol. Environ. Chem. 1996, 54, 23.

51. Pfeiffer, W. C.; Trindade, H. A.; Londres, H.; Costa-Ribeiro, C. L.; Environm. Sci. Technol. 1981, 15, 84.

52. Botkin, D. B.; Keller, E. A.; Environmental Science, $2^{\mathrm{a}}$ ed., John Wiley \& Sons, Inc.; New York, 1998; p 477.

53. Anfossi, D.; Sandroni, S.; Atmos. Environ. 1997, 31, 3481.

54. Baird, C.; Environmental Chemistry, $2^{\mathrm{a}}$ ed., W. H. Freeman and Company: New York, 1995, p. 82.

55. CETESB. Qualidade do ar no Estado de São Paulo - 1989. São Paulo, 1990.

56. Miguel, A. H.; Quim. Nova 1992, 15, 118.

57. IUPAC Solubility Data Series, Battino, R., ed.; Pergamon Press: Oxford, 1981, vol. 7.

58. Kirk-Othmer; Encyclopedia of Chemical Technology, $2^{\text {nd }}$. ed., J. Wiley: New York, 1967, vol. 14, p. 412.

59. Book of Tables Chemistry, $9^{\text {th }}$. ed., VEB Deutscher Verlag für grundstoffindustrie: Leipzig, 1982.

60. Aylward, G. H.; Findlay, T. J.V.; Summary of data chemistry in SI units, 2, Auflage, Verlag Chemie: Weinheim, 1981.

61. IUPAC Solubility Data Series, Young, C. L., ed., Pergamon Press: Oxford, 1981, vol. 8.

62. Waldmann, K. Z.; Anorg. Allg. Chem. 1955, 281,135.

63. Guthrie, J. P.; Can. J. Chem. 1979, 57, 454.

64. Baird, N. C.; Taylor, K. F.; J. Comput. Chem. 1981, 2, 225.

65. Golding, R. M.; J. Chem. Soc. 1960, 3711.

66. Gouger, S.; Stuehr, J.; Inorg. Chem. 1974, 13, 379.

67. Fábián, I.; Gordon, G.; Inorg. Chem. 1991, 30, 3994.

68. Lutz, B.; Wendt, H.; Ber. Bunsen-Ges. Phys. Chem. 1970, 74, 372.

69. Bottero,J. -Y.; Manceau, A.; Viellieras, F.; Tchoubar, D.; Langmuir 1994, $10,316$.

70. Xue, H.; Gonçalves, M. S.; Reutlinger, M.; Sigg, L.; Stumm, W.; Environ. Sci. Technol. 1991, 25, 1716.

71. Weschler, C. J.; Mandich, M. L.; Graedel, T. E.; J. Geophys. Res. 1986, 1, 5789.

72. Hoffmann, H.; Hofmann, P.; Lieser, K. H.; Fresenius' J. Anal. Chem. 1991, 340,591 .

73. Wilkinson, P. M.; Dolderssum, B.; Cramers, P. H.; van Dierendonck, L. L.; Chem. Eng. Sci. 1993, 48, 933.

74. Cotton, F. A.; Wilkinson, G.; Química Inorgânica, $1^{\text {a }}$ ed., Livros Técnicos e Científicos Editora S.A.: Rio de Janeiro, 1978, p. 316.

75. Loomis, A. G. Em International Critical Tables, McGraw-Hill: New York, 1928, vol. III, p. 255-261.

76. Schwartz, S. E.; Whitr, W. H.; Adv.Environ. Sci. Technol. 1983, 12, 1.

77. Briner, E.; Perrotet, E.; Helv. Chim. Acta 1939, 22, 397.

78. Rand, M. C.; Gale, S. B. Em Principles and Applications of Water Chemistry, Proceedings of the Fourth Rudolfs Research Conference, Rutgers, The State University, New Brunswick, NJ; Faust, S. D.; Hunter, J. H., eds.; J. Wiley \& Sons, Inc.: New York, 1967, pp. 380-404.

79. Matsuura, A.; Harada, J.; Akehata, T.; Shirai, T.; J. Chem. Eng. Jpn. 1969, $2,199$.

80. Fedorova, S. K.; Skibida, I. P.; Gladikii, A.V.; J. Appl. Chem. USRR 1976, 49, 753.

81. Larson, T. V.; Horike, N. R.; Harrison, H.; Atmos. Environ. 1978, 12, 1597.

82. Penket, S. A.; Jones, B. M. R.; Brice, K. A.; Eggleton, A. E. J.; Atmos. Environ. 1979, 13, 123.

83. Brandt, C.; Coichev, N.;Hostert, E.; van Eldik, R.; GIT 1993, 37, 277. 
84. Braga, T. G.; Connick, R. E. Em Flue Gas Desulfurization; Hudson, J. L.; Rochelle, G. T., eds., ACS Symposium Series 188; American Chemical Society: Washington, DC, 1982, pp. 153-171.

85. Cladkii, A. V.; J. Anal. Chem. USSR 1984, 57, 1964.

86. Hegg, D. A; Hobbs, P. V.; Atmos. Environ. 1978, 12, 241.

87. Martins, C. R.; Neto, C. A.C.; Alves, J. J. F.; de Andrade, J. B.; J. Braz. Chem. Soc. 1999, 10, 453.

88. Brandt, C.; Elding, L. I.; Atmos. Environ. 1998, 32, 797.

89. Pirt, S. J.; Callow, D. S.; Gillett, W. A.; Chem. Ind. 1957, 730.

90. Junge, C. E.; Ryan, T. G.; Q. J. R. Met. Soc. 1958, 84, 46.

91. Lunák, S,; El Wakil, A. M.; Veprek-Siska, J.; Collect. Czech. Chem. Commun. 1978, 43, 3306.

92. Backström, H.J.; Z. Phys. Chem. 1934, 25B, 122

93. Bassett, H.; Parker, W. A.; J. Chem. Soc. 1951, 1540.

94. Freiberg, J.; Atmos. Environ. 1975, 9, 661.

95. Sung, W.; Morgan, J. J.; Environ. Sci. Technol. 1980, 14, 561.

96. Klug-Roth, D.; Rabani, J.; J. Phys. Chem. 1976, 80, 588.

97. Ibusuki, T.; Takeuchi, K.; Atmos. Environ. 1987, 21, 1555.

98. Conklin, M. H.; Hoffmann, M. R.; Environ. Sci. Technol. 1988, 22, 891.

99. Anast., J. M.; Margerum, D. W.; Inorg. Chem. 1981, 20, 2319.

100. Ali, M.; Sasha, S. K.; Banerjee, P.; Bull. Chem. Soc. Jpn. 1991, 64, 2497.

101. Sato, T.; Okabe, T.; Nippon Kagaku Kaishi 1977, 1124.

102. Cavicchi, G.; Ann. Chim.(Rome) 1951, 41, 411.

103. Kraft, J.; Ph. D. Thesis; University of Frankfurt, Main, Germany, 1987.

104. Faust, B. C.; Hoffmann, M. R.; Bahnemann, D. W.; J. Phys. Chem. 1989, 93, 6371.

105. Brandt, C.; van Eldik, R.; Atmos. Environ. 1997, 24, 4247.

106. Veprek- Siska, J.; Wagnerová, D. M.; Eckschlager, K.; Collect. Czech. Chem. Commun. 1996, 31, 1248.

107. Oblath,S. B.; Markowitz, S. S.; Novakov, T.; Chang, S. G.; J. Phys. Chem. 1981, 85,1017 .
108. Coichev, N.; van Eldik, R.; New J. Chem. 1994, 18, 123.

109. Littlejohn, D.; Wang, Y.; Chang, S.; Environ. Sci. Technol. 1993, 27, 2162.

110. Martin, , L. R.; Damschen, D. E.; Judeikis, H. S.; Atmos. Environ. 1981, 15,191

111. Ellison, T. K.; Eckert, C. A.; J. Phys. Chem. 1984, 88, 2335.

112. de Andrade, J. B.; Sarno, P.; Quim. Nova 1990, 13, 213.

113. de Andrade, J. B.; Aragão, N. M.; Araújo, F. R. J.; Quim. Nova 1995, 18,5.

114. Sakugawa, H.; Kaplan, I. R.; Shepard, L.; Atmos. Environ. 1993, 27 B, 203.

115. Galloway, J. N.; Likens, G. E.; Keene, W. C.; Miller, J. M.; J. Geophys. Res. 1982, 87, 8771.

116. Galloway, J. N.; Likens, G. E.; Atmos. Environ. 1981, 15, 1081.

117. Andreae, M. O.; Talbot, R. W.; Andreae, T. W.; Harris, R. C.; J. Geophys. Res. 1988, 93, 1616.

118. Fowler, D.; Cape, J. N.; Leith, I. D.; Paterson, I. S.; Kinnaird, J. W.; Nicholson, I. A.; Nature 1982, 297, 383

119. Miguel, A. H.; Pomaleski, D. R. M.; Schilling, M.; Klockow, D.; Carvalho, C. R.; Resumos da $13^{a}$ Reunião Anual da Sociedade Brasileira de Química, Caxambu, Brasil, 1990.

120. Souza, S. R.; Carvalho, L. R. F.; Quim. Nova 1997, 20, 245.

121. Waldman, J. M.; Munger, J. W.; Jacob, D. J.; Flagan, R. C.; Morgan, J. J.; Hoffmann, M. R.; Science 1982, 218, 677.

122. Galloway, J. N.; Linkens, G. E.; Edgerton, E. S.; Science 1976, 194, 722

123. Glavas, S.; Atmos Environ. 1988, 22, 1505.

124. Khemani, L. T.; Indian J. Radio Space Phys. 1993, 22, 207.

125. Campos, V. P.; Tese de Doutoramento, Universidade Federal da Bahia, Brasil, 1995.

126. de Andrade, J. B.; Pinheiro, H. C.; Andrade, M. V.; J. Braz. Chem. Soc. $1995,3,287$.

127. Oliveira, A. E.; de Andrade, J. B.; Quim.Nova 1994, 17, 13.

128. de Andrade, J. B.; Tanner, R. L.; Atmos. Environ. 1992, 26, 819.

129. Hering, S. V.; Friedlander, S. K.; Atmos, Environ. 1982, 16, 2647. 